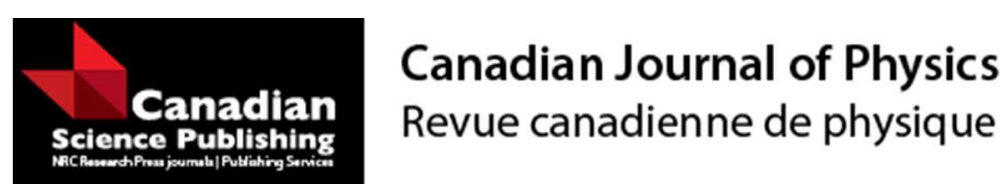

Transient flows past arrays of yawed finite prisms

\begin{tabular}{|r|l|}
\hline Journal: & Canadian Journal of Physics \\
\hline Manuscript ID & cjp-2017-0057.R1 \\
\hline Manuscript Type: & Article \\
\hline Date Submitted by the Author: & 06-Apr-2017 \\
\hline Complete List of Authors: & $\begin{array}{l}\text { Liu, I-Han; Lehigh University } \\
\text { Attiya, Bashar; Lehigh University } \\
\text { Oztekin, Alparslan; Lehigh University, Dept.of Mechanical Engineering \& } \\
\text { Mechanics; Lehigh University }\end{array}$ \\
\hline Keyword: & $\begin{array}{l}\text { large eddy simulations, drag coefficient, tandem and staggered arrays, } \\
\text { turbulent wake flows, yawed prisms }\end{array}$ \\
\hline $\begin{array}{r}\text { Is the invited manuscript for } \\
\text { consideration in a Special } \\
\text { Issue? : }\end{array}$ & N/A \\
\hline
\end{tabular}




\title{
1 Transient flows past arrays of yawed finite prisms
}

2
3
4
5
6

\author{
I-HAN LIU \\ BASHAR ATTIYA \\ ih1211@1ehigh.edu \\ bha212@1ehigh.edu \\ ALPARSLAN OZTEKIN* \\ alo2@1ehigh.edu \\ P.C. Rossin College of Engineering and Applied Science, Lehigh University, Bethlehem, PA 18015, USA \\ * Corresponding author
}

7 Abstract

8 Transient flows past arrays of yawed finite length prisms in tandem and staggered 9 arrangements are simulated. Large Eddy Simulations (LES) method is employed to characterize

10 spatial and temporal characteristics of flows in the vicinity of prisms. Drag coefficients of prisms

11 are determined for different spacing and yaw angle of prisms. Three-dimensional effects are

12 investigated for different spacing of prisms in a tandem array. Drag coefficient of downstream

13 prism is significantly lower compared to that of upstream prism when prisms are tightly spaced.

14 Drag coefficient of downstream prism predicted by simulations in three-dimensional geometry is

15 recovered fully as spacing approaches ten times of the height of the prism while drag coefficient

16 predicted by simulations in two-dimensional geometry is only about $30 \%$ of the upstream prism

17 even when two prisms are separated by twenty times the height of the prism. The wake flow

18 patterns do not influence the drag coefficient strongly for the staggered arrays of yawed prisms.

19 Prisms can be placed in a tighter arrangement without a significant penalty of drag reduction.

20 Mathematical models and numerical methods employed are validated by comparing simulation

21 results against experimental results. Drag exerted on these prisms can directly be related to

22 power generation by devices containing translating blades of marine-current applications. This

23 study demonstrates that arrangement, spacing and yaw angle can be used to design and optimize

24 energy harvesting devices and other offshore structures.

25 Keywords

26 large eddy simulations; drag coefficient; tandem and staggered arrays; yawed prisms; turbulent

27 wake flows 


\section{Introduction}

Flows past multiple bluff bodies are encountered in many engineering applications, such as chimney stacks, buildings, bridges, and offshore structures. Offshore structures like marine

31 current energy harvesting devices are often placed in multiple units with an array configuration.

32 Turbulent wake flows generated by upstream units will increase loading of downstream units and

33 thus can compromise the durability of structures. Understanding the solid-fluid interactions in

34 flows past structures is important to design and optimize marine current devices.

Flows past a single infinite prism have been well studied both experimentally and numerically [1-9]. When multiple prisms are in the field with close proximity of each other, flow

37 structures become more complex due to the interactions between wake flows and prisms. Prisms

38 situated downstream interfere with the wake flow structure induced by upstream prisms. This

39 interaction has profound influence on the drag and the lift forces exerted on prisms. Flows past

40 multiple prisms in tandem arrangement are classical flow problems in engineering applications

41 and have been studied by many investigators [6,10-12].

It is well-documented that flow patterns are strongly dependent on the aspect ratio, $G / D$,

43 where $G$ is the gap between two successive infinite prisms and $D$ is the diameter of the prism.

44 Different flow regimes are encountered depending on values of $G / D$. Three types of wake

45 behavior are classified [13-15]. At low values of $G / D$, the vortex shedding from upstream prism

46 is suppressed. Two prisms act like a single body since the shear layer separated from upstream

47 prism wraps around the downstream prism. At intermediate values of $G / D$, the vortex shedding

48 from downstream prism synchronized with shedding from the upstream prism. At large values of

$49 G / D$, the Karman vortex street generated from both upstream and downstream prisms does

50 hardly interfere with each other. As described in Auteri et al. [15], critical values of $G / D$ can be 
51 identified as wake flow transitions from one pattern to another. Drag and lift coefficient and

52 Strouhal number display rapid changes at around the critical values of $G / D$, as reported in

53 Sakamoto et al. [6] and Liu and Chen [10]. Several investigators have reported hydrodynamic

54 loads in flows past arrays of tandem cylinders for various values of $G / D$. An extensive review on

55 this topic is given by Sumner [13]. For low and intermediate values of $G / D, C_{D}$ of the upstream

56 cylinder in an array is lower than $C_{D}$ of the single cylinder at the same $\operatorname{Re} . C_{D}$ of the upstream

57 cylinder decreases as $G / D$ is increased. At values of $G / D$ near criticality, $C_{D}$ of the upstream

58 prism increases rapidly. Sudden increase in $C_{D}$ coincides with wake flow pattern changes

59 induced by the Karman vortex shedding. Vortex shedding set off from both upstream and

60 downstream prisms for $G / D$ around the critical value. As $G / D$ is increased beyond the critical

61 value, $C_{D}$ gradually approaches the value of $C_{D}$ in flows past a single prism. Generally speaking,

$62 C_{D}$ of the downstream prism is lower than that of the upstream prism due to wake effects [12].

Aforementioned studies consider prisms whose axes are perpendicular to the oncoming

64 flow. Flows past an infinite yawed prism are also well-documented. Shirakashi et al. [16]

65 conducted experiments in flows past a yawed cylinder, and reported that the vortex shedding

66 become irregular and the vortex shedding frequency is lower for the yawed cylinder compare to

67 that for the perpendicular cylinder. Numerical investigations for flows past an inclined cylinder

68 reported by Yeo and Jones [17] concluded the swirling flow around the cylinder is responsible

69 for generating low-frequency loadings. Zhao et al. [18] performed direct numerical simulation to

70 study the effects of the yaw angle on wake structures, vortex shedding frequencies, and

71 hydrodynamic forces. They have documented that vortices shed from the cylinder propagate

72 along the axis of the cylinder axis while they are convected downstream. They also reported that

73 the drag coefficient increases gradually as the incline angle is increased. The yaw angle in these 
74 studies is defined as the angle between the oncoming flow direction and the direction 75 perpendicular to the axis of the prism. Jordan [19] investigated flow past yawed cylinders at the 76 angle of $45^{\circ}$ and $60^{\circ}$ as referenced to the flow direction. Jordan [19] characterized the flow 77 transition to turbulence in the wake of yawed cylinders.

Despite of extensive studies in flow past infinite prisms (cylinders), many of actual

79 structures should be modeled as a finite rectangular prism with free ends. Several investigators

80 studied flows past a finite length prism or a cylinder with one free end [20,21]. Sumner [22],

81 recently reported a comprehensive review on the subject. To date, there are not many

82 investigations considering flows past multiple bluff bodies with two free ends.

Flow patterns in the wake of finite prisms with free ends are fully three-dimensional. The

84 wake flow field is characterized by both the alternating Karman vortex shedding that occurs

85 along the prism, and the tip vortices formed at free ends [23]. Wang et al. [20] experimentally

86 studied interactions between the boundary layer and the wake of wall-mounted finite square

87 prism. They concluded that highly three-dimensional flow patterns around a finite square prism

88 induced by the Karman vortex along the surface of the prism and tip and base vortices within the

89 wake. Rostamy et al. [24] conducted experiments for finite square prisms of various length to

90 diameter $(L / D)$ ratios. Their results showed aspect ratio has profound influence in the velocity

91 field around free ends. The wake is dominated by tip vortices at lower values of $L / D$, while for

92 higher values of $L / D$, the Karman vortex shedding from the prism sides dominates the wake flow 93 pattern.

94 The drag coefficient of finite length cylinder for different values of $L / D$ has been reported 95 extensively. $C_{D}$ is decreased as $L / D$ is decreased [21,25-27]. Zdravkovich et al. [25] carried out 
96 experiments on a finite length circular cylinder with two free ends for various values of $L / D$. It is

97 observed that $C_{D}$ decreases with decreasing $L / D$. Inflow around cylinder ends causes an increase

98 of pressure at the back side of the cylinder, which in turn reduces the drag coefficient. Strouhal

99 number $(S t)$ varies with $L / D$. Vortex shedding behind the prism becomes more irregular due to

100 the presence of unstable tip vortices. Wang and Zhou [27] experimentally studied the near wake

101 of a finite length square cylinder. They reported that the spatial wake configuration is dependent

102 on the interaction between the Karman vortex shedding (asymmetric flow pattern) and the tip

103 vortices (symmetric flow pattern). As $L / D$ ratio is decreased, tip vortices dominate wake flow

104 patterns. Symmetrical vortex patterns correspond to weaker stresses, which lead to lower $C_{D}$

105 compared against infinitely long cylinders. They concluded that the presence of tip vortices

106 contributes to the decrease of $C_{D}$ monotonically with a decrease of $L / D$. There are only few

107 studies for flows past finite yawed prisms. As reported by $\mathrm{Hu}$ et al. [28], the flow field in the

108 vicinity of a finite prism differs from that of an infinite prism. LES simulations presented by $\mathrm{Hu}$

109 et al. [28] revealed that the drag coefficient of the finite yawed prism decreased as the yaw angle

110 (as specified an angle from the vertical axis) is increased. Down-wash and up-wash flows behind

111 the yawed cylinder resulted in downward and upward axial flows. Vortex pairs at the free end

112 also cause a decrease in the shedding frequency.

113 The present study focuses on the characterization of transient flows past arrays of yawed

114 finite prisms in different arrangements. Simulations employing the LES model are carried out to 115 study wake flow patterns and their influence on drag coefficients in flows past a single and 116 multiple finite length prisms with two free ends. Drag coefficient of prisms in various arrays is 117 presented. The aspect ratio, $G / D$, and the yaw angle are varied and their influence on the flow 118 structures is presented. To the best knowledge of present authors, flows past arrays of yawed 
119 finite prisms with tandem and staggered arrangements have not been considered previously. The

120 present study can aid designing and optimizing marine current energy harvesting devices by

121 characterizing wake flow patterns and their influence on the forces exerted on structures.

\begin{tabular}{|llll|}
\hline NOMENCLATURE & & \\
$A$ & projection area & $\Delta t$ & time step size \\
$C_{D}$ & drag coefficient & $u_{i}, u_{j}$ & velocity \\
$C_{L}$ & lift coefficient & $\bar{u}_{i}, \bar{u}_{j}$ & resolved scale velocity vector \\
$C_{p}$ & pressure coefficient & $U_{\infty}$ & free stream velocity \\
$C_{w}$ & WALE constant & $V$ & volume of cell \\
$D$ & prism height & $w$ & prism width \\
$d$ & tip to the centerline distance & $\omega$ & vorticity magnitude \\
$F_{D}$ & drag force & $x_{i}, x_{j}$ & position vector \\
$F_{L}$ & lift force & $y$ & distance to the nearest wall \\
$f$ & vortex shedding frequency & & \\
$G$ & spacing distance & $\mathbf{G R E E K ~ S Y M B O L S}$ \\
$h$ & square cylinder height & $\lambda$ & non-dimensional time \\
$L$ & prism length & $\Delta \lambda$ & non-dimensional time step \\
$L_{S}$ & SGS mixing length & $\theta$ & yaw angle \\
$m_{1}, m_{2}, m_{3}$ & number of cells & $\delta_{i j}$ & Kronecker delta \\
$\bar{p}$ & resolved pressure & $\mu$ & dynamic viscosity \\
$p_{\infty}$ & reference pressure & $v$ & kinematic viscosity \\
$R e$ & Reynolds number & $v_{t}$ & SGS eddy viscosity \\
SGS & subgrid scale & $\rho$ & Density \\
$S t$ & Strouhal number & $\kappa$ & von Karman constant \\
$\bar{S}_{i j}$ & rate-of-strain tensor & $\tau_{i j}$ & subgrid stress tensor \\
$t$ & time & & \\
\hline
\end{tabular}

\section{Mathematical Model}

124 Large eddy simulation approach applies a spatial filtering to the Navier-Stokes equation.

125 The large-scale eddies are resolved numerically and the smaller-scale eddies - eddies that are

126 smaller than the grid size - are not resolved but are approximated. Resolving the large-scale 
127 motions enables LES to predict instantaneous flow characteristics and to resolve turbulent flow 128 structures.

129 Time and spatial averaged Navier-Stokes equations with low pass filtering yield

$130 \quad \frac{\partial \bar{u}_{j}}{\partial x_{j}}=0$

$131 \frac{\partial \bar{u}_{i}}{\partial t}+\frac{\partial \bar{u}_{j} \bar{u}_{i}}{\partial x_{j}}=-\frac{1}{\rho} \frac{\partial \bar{p}}{\partial x_{i}}-\frac{\partial \tau_{i j}}{\partial x_{j}}+v \frac{\partial^{2} \bar{u}_{i}}{\partial x_{j} \partial x_{j}}$

132 where $\bar{u}_{j}=u_{j}-\hat{u}_{j}$ represents the filtered velocity vector and the over bar denotes filtering

133 operator; $\rho$ is the fluid density; and $\bar{p}$ is the resolved pressure.

134 The subgrid shear stress $\tau_{i j}=\overline{u_{i} u_{j}}-\bar{u}_{j} \bar{u}_{i}$ is calculated using the subgrid-scale (SGS)

135 models. Employing the Boussinesq hypothesis the SGS turbulent stress is computed from

$136 \tau_{i j}-\frac{1}{3} \tau_{k k} \delta_{i j}=-2 v_{t} \bar{S}_{i j}$

137 where $\delta_{i j}$ is the Kronecker delta; $v_{t}$ is the SGS eddy viscosity; and $\bar{S}_{i j}=\frac{1}{2}\left(\frac{\partial \bar{u}_{i}}{\partial x_{j}}+\frac{\partial \bar{u}_{j}}{\partial x_{i}}\right)$ is the 138 rate-of-strain tensor.

139 The wall-adapting local eddy-viscosity (WALE) method is used to model the SGS eddy-

140 viscosity [29]:

$141 \quad \boldsymbol{v}_{t}=\boldsymbol{L}_{s}^{2} \frac{\left(s_{i j}^{d} s_{i j}^{d}\right)^{3 / 2}}{\left(\bar{s}_{i j} \bar{s}_{i j}\right)^{5 / 2}+\left(s_{i j}^{d} s_{i j}^{d}\right)^{5 / 4}}$

$142 S_{i j}^{d}=\frac{1}{2}\left(\left(\frac{\partial \bar{u}_{i}}{\partial x_{j}}\right)^{2}+\left(\frac{\partial \bar{u}_{j}}{\partial x_{i}}\right)^{2}\right)-\frac{1}{3} \delta_{i j}\left(\frac{\partial \bar{u}_{k}}{\partial x_{k}}\right)^{2}$

$143 \quad L_{s}=\min \left(\kappa y, C_{w} \mathrm{~V}^{1 / 3}\right)$ 
144 Here $L_{s}$ is the mixing length for subgrid scales; $\kappa$ is the von Karman constant; $y$ is the shortest 145 distance to the wall; the WALE constant $C_{w}$ is 0.325 ; and $V$ is the mesh cell volume. Details of 146 the WALE model are documented by Nicoud and Ducros [29].

\section{Computational domains}

LES simulations for flows past prisms are conducted at $R e=50,000$. Reynolds number is

149 defined as $\operatorname{Re}=\rho U_{\infty} D / \mu$, where $U_{\infty}$ is the free stream velocity, $D$ is the prism height, and $\mu$ is the

150 fluid dynamic viscosity. The schematic of the three-dimensional computational domain is shown

151 in Fig. 1(a)-Fig. 1(c) while the top view ( $x-z$ plane) are shown in Fig. 1(d)-Fig. 1(e). Fig. 1(a)-

152 Fig. 1(d) shows prisms that are arranged in a tandem array and situated perpendicular to the

153 oncoming flow. Fig. 1(c)-Fig. 1(e) shows prisms that are arranged in a staggered array and

154 placed against the oncoming flow with a yaw angle, $\theta$. The yaw angle in the present study is

155 defined as the angle between the axis of prism and the oncoming flow direction. The geometry of

156 a single prism is depicted in Fig. 1(b). The length, height, and width of the prism are $L, D$, and $w$,

157 respectively. Spacing between two successive prisms, $G$, and the yaw angle, $\theta$, are illustrated in

158 Fig. 1. For staggered arrays, distance from prism tip to the centerline is defined as $e$. Simulations

159 for flows past staggered arrays of prisms are conducted for fixed value of $e$. Prisms in tandem

160 arrays are labeled as Pi while prisms in staggered arrays are labeled as Ai. Prisms are numbered

161 in an order from upstream to downstream. Values of physical properties and geometric and flow

162 parameters used in the present simulations are listed in Table 1. Table 2 lists different prism

163 array configurations. Each configuration has different spacing $G$, yaw angle, $\theta$, and number of

164 prisms in the arrangement. 


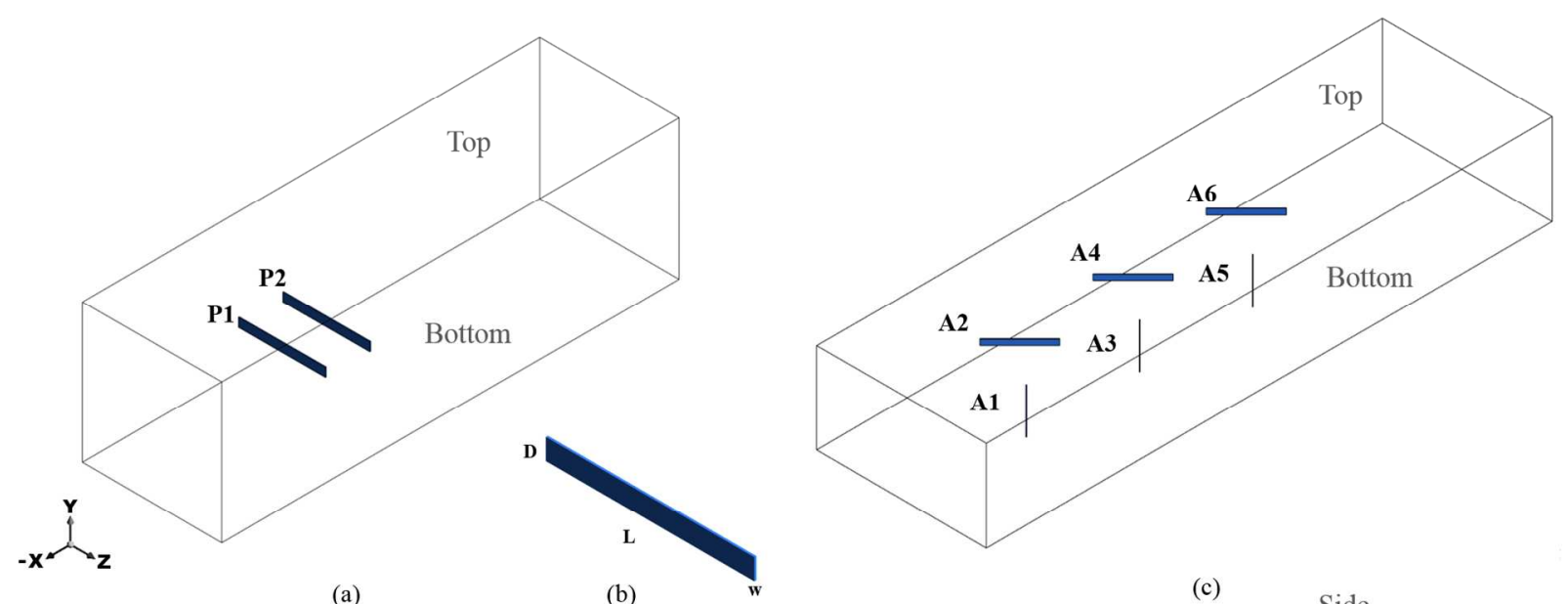

(a)

(b)

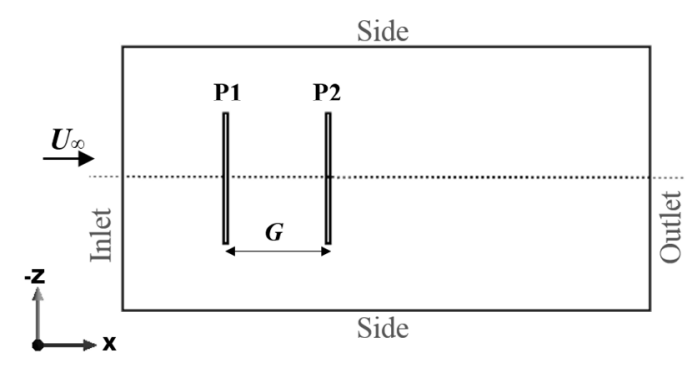

(d)

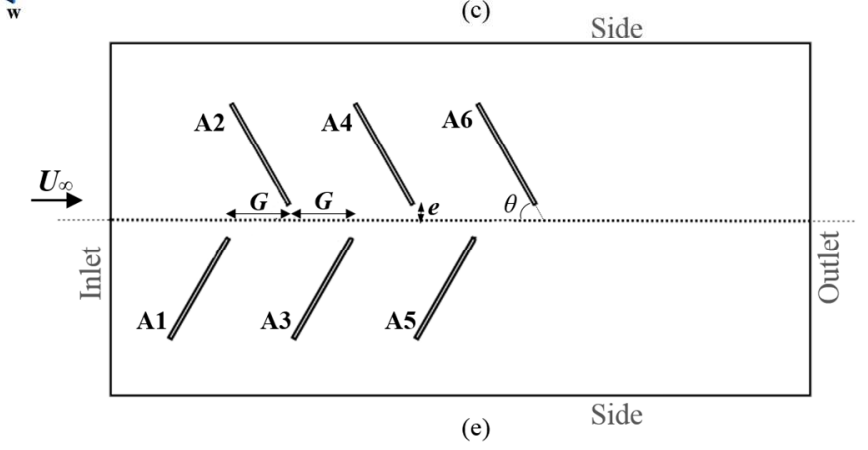

\section{6}

167

168

169

\section{0}

Table 1. Parameters used in the present study.

\begin{tabular}{|lrllrl|}
\hline \multicolumn{7}{|l|}{ Parameter Values } \\
$D$ & 0.1 & {$[\mathrm{~m}]$} & $U_{\infty}$ & 0.5 & {$\left[\mathrm{~m} \mathrm{~s}^{-1}\right]$} \\
$e$ & 0.2 & {$[\mathrm{~m}]$} & $w$ & 0.01 & {$[\mathrm{~m}]$} \\
$L$ & 1 & {$[\mathrm{~m}]$} & $\rho$ & 1000 & {$\left[\mathrm{~kg} \mathrm{~m}^{-3}\right]$} \\
$\operatorname{Re}$ & 50,000 & {$[-]$} & $\mu$ & 0.001 & {$\left[\mathrm{~kg} \mathrm{~m}^{-1} \mathrm{~s}^{-1}\right]$} \\
\hline
\end{tabular}

171

172 Table 2. Various array arrangements of prisms.

\begin{tabular}{|c|c|c|cccccccc|}
\hline & Spacing & Angle & \multicolumn{10}{|c|}{ Prism label } \\
\hline & $\boldsymbol{G}$ & $\boldsymbol{\theta}$ & P1 & P2 & A1 & A2 & A3 & A4 & A5 & A6 \\
\hline \multicolumn{1}{|c|}{ Single prism in different angles } \\
\hline Case 1 & - & $45^{\circ}$ & - & - & $\vee$ & - & - & - & - & - \\
Case 2 & - & $60^{\circ}$ & - & - & $\vee$ & - & - & - & - & - \\
Case 3 & - & $90^{\circ}$ & - & - & $\vee$ & - & - & - & - & - \\
\hline
\end{tabular}




\begin{tabular}{|c|c|c|c|c|c|c|c|c|c|c|}
\hline \multicolumn{11}{|c|}{ Two tandem prisms with different gaps } \\
\hline Case 4 & $5 D$ & $90^{\circ}$ & V & V & - & - & - & - & - & - \\
\hline Case 5 & $7 D$ & $90^{\circ}$ & V & V & - & - & - & - & - & - \\
\hline Case 6 & $10 D$ & $90^{\circ}$ & V & V & - & - & - & - & - & - \\
\hline Case 7 & $20 D$ & $90^{\circ}$ & V & $\vee$ & - & - & - & - & - & - \\
\hline \multicolumn{11}{|c|}{ Multiple staggered prisms with different gaps and angles } \\
\hline Case 8 & $10 D$ & $45^{\circ}$ & - & - & $\overline{\mathrm{V}}$ & $\vee$ & - & - & - & - \\
\hline Case 9 & $10 D$ & $60^{\circ}$ & - & - & V & V & - & - & - & - \\
\hline Case 10 & $10 D$ & $45^{\circ}$ & - & - & V & V & V & V & - & - \\
\hline Case 11 & $2.5 D$ & $45^{\circ}$ & - & - & V & V & V & V & - & - \\
\hline Case 12 & $10 D$ & $60^{\circ}$ & - & - & V & V & V & V & - & - \\
\hline Case 13 & $5 D$ & $45^{\circ}$ & - & - & V & V & V & V & - & - \\
\hline Case 14 & $10 D$ & $45^{\circ}$ & - & - & V & V & V & V & V & V \\
\hline
\end{tabular}

175 examples: dimensions of the domain for case 5 is $52.5 D \times 16 D \times 16 D$ while dimensions for case

17611 is $60 D \times 16 D \times 30 D$ in the stream-wise, the cross stream, and the span-wise direction,

177 respectively. For all cases, the length of the inlet region - distance from the inlet to the closest

178 upstream prism - is selected as $12 \mathrm{D}$ and the distance from the outlet to the closest downstream

179 prism is selected as $30 D$ to minimize the influence of the imposed inlet and the outlet velocity

180 boundary conditions on the flow field near prisms.

\section{Numerical Methods, Convergence and Validation Studies}

ANSYS FLUENT commercial software is utilized to conduct simulations. The semi-

183 implicit method for pressure-linked equations (SIMPLE) solution algorithm that fully couples

184 the velocity and the pressure field is employed to solve the mathematical model. The bounded 185 central differencing and bounded second order implicit scheme are used for discretizing 186 momentum equation and time, respectively. Detailed descriptions of numerical schemes and 187 simulation tools are provided in ANSYS theory guide and ANSYS user guide [30,31]. 
189 rate is prescribed at the inlet while zero gauge pressure is applied at the outlet. No-slip and no190 penetration boundary conditions are imposed along surfaces of prisms and top and bottom 191 surfaces of the channel. Symmetry boundary conditions are imposed on the side surfaces of the 192 computational domain.

OpenFOAM, an open source CFD software package, is used here to validate the

194 numerical model implemented in ANSYS FLUENT. Flow geometry depicted in case 3 and case 1955 are considered for validation study. The "pimpleFoam" solver that combines the Pressure196 Implicit with Splitting of Operators (PISO) and SIMPLE algorithms are employed. An adjustable 197 time step function with a constraint in Courant number in pimpleFoam solver is employed to 198 enhance numerical stability. Details of the methods are documented by Greenshields [32].

\section{Spatial and Temporal Convergence}

Mesh structures for cases 5 and 11 are shown in Fig. 2. Finer mesh is used in regions near

201 the surface of prisms to capture boundary layer separations and to resolve near wake flow 202 patterns. Mesh becomes gradually coarser away from prisms, as depicted in Fig. 2. A mesh 203 optimization study is conducted for case 5 . Meshes of different density generated are: $1.2 \times 10^{6}$ 204 elements $\left(\mathrm{m}_{1}\right), 2.4 \times 10^{6}$ elements $\left(\mathrm{m}_{2}\right)$, and $5.7 \times 10^{6}$ elements $\left(\mathrm{m}_{3}\right)$. The main difference between 205 mesh $\mathrm{m}_{1}$ and mesh $\mathrm{m}_{2}$ is an increase of number of cells in the region near prisms. The average

206 value of drag coefficient for each prism in the array is calculated for $\mathrm{m}_{1}, \mathrm{~m}_{2}$ and $\mathrm{m}_{3}$. Drag

207 coefficient is defined by $C_{D}=F_{D} /\left(\frac{1}{2} \rho U_{\infty}^{2} A\right)$, where $F_{D}$ is the drag force exerted by fluid on the 208 prism and $A$ is the area of the prism projected perpendicular to the oncoming flow. Non- 
209 dimensional time and non-dimensional time step are defined as $\lambda=t U_{\infty} / D$ and $\Delta \lambda=\Delta t U_{\infty} / D$, 210 respectively.
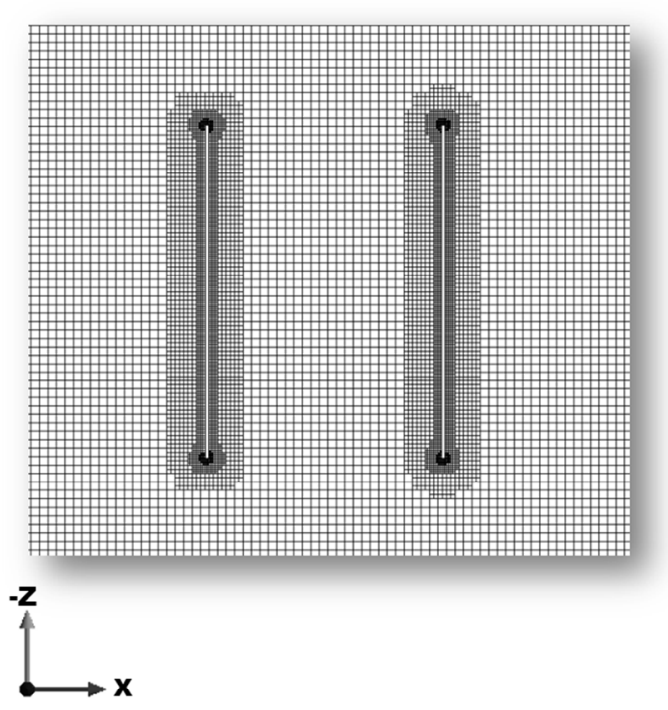

211

212

213

214

215

216

217

(a) plane.

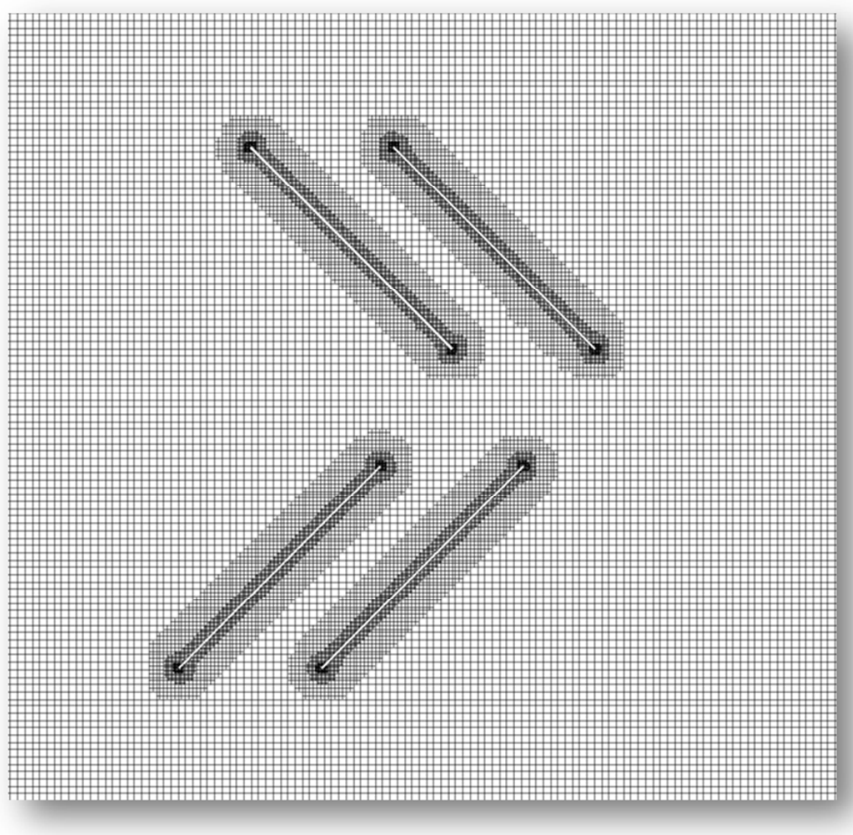

(b)

Fig. 2. Examples of mesh around prisms for (a) case 5 and (b) case 11 . Images are taken at $y=0$

The drag coefficient calculated by using FLUENT and OpenFOAM solver for case 5 is shown in Fig. 3 as a function of time. $\Delta \lambda=0.004$ is used for simulations conducted with FLUENT while adjustable time step is used for simulations conducted with OpenFOAM. Graphs on the left column denote drag coefficient of the upstream prism P1 and graphs on the right column denote drag coefficient of the downstream prism P2. Rows from top to bottom denote

219 time signature of drag coefficient obtained using $\mathrm{m}_{1}, \mathrm{~m}_{2}$ and $\mathrm{m}_{3} \mathrm{mesh}$, respectively. The last two 220 rows show drag coefficients predicted by OpenFOAM solver using $\mathrm{m}_{1}$ and $\mathrm{m}_{3}$ mesh density.

221 Presence of irregular fluctuations in drag coefficient signal, as shown in Fig. 3(a) and Fig. 3(d), 222 indicates alternating flow patterns in the wake region. Similar characteristics have been reported 
223 in flows past a normal prism [5,33]. In order to demonstrate if these flow patterns alternate

224 periodically or irregularly simulations needs to be carried out with much larger flow times. Due

225 to limited computational resources present simulations are halted at $\lambda=700$. High frequency

226 fluctuations are detected in the signature of $C_{D}$ for $\mathrm{P} 2$. This is due to the fact that oncoming flows

227 for P2 are highly turbulent wake flows induced by the presence of P1 in the field.

(a)

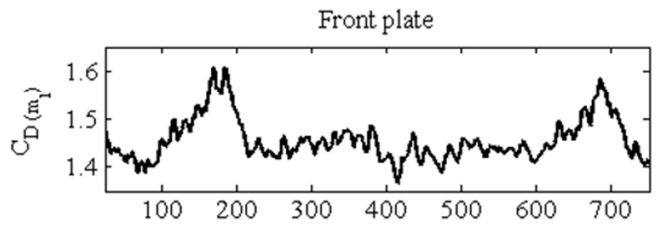

(b)

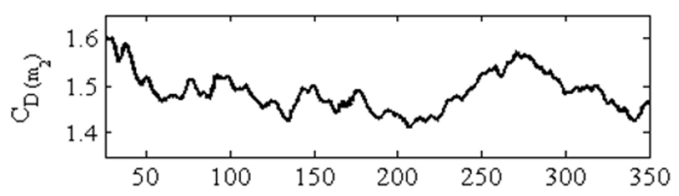

(c)

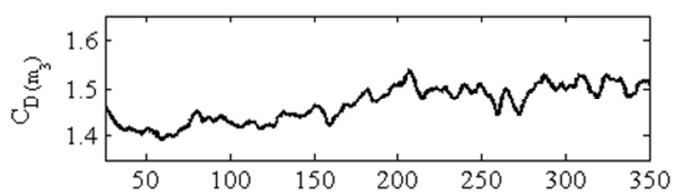

(d)

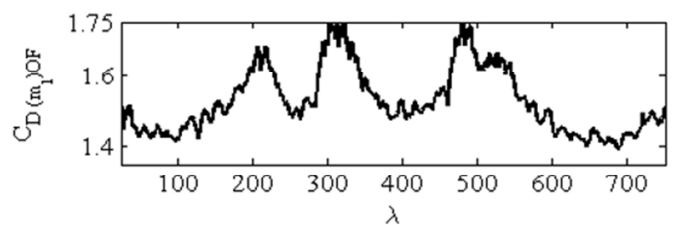

(e)

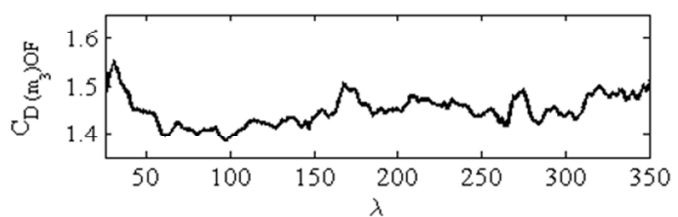

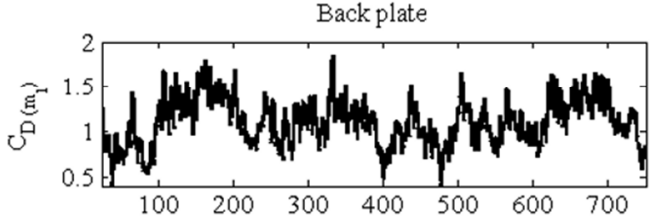
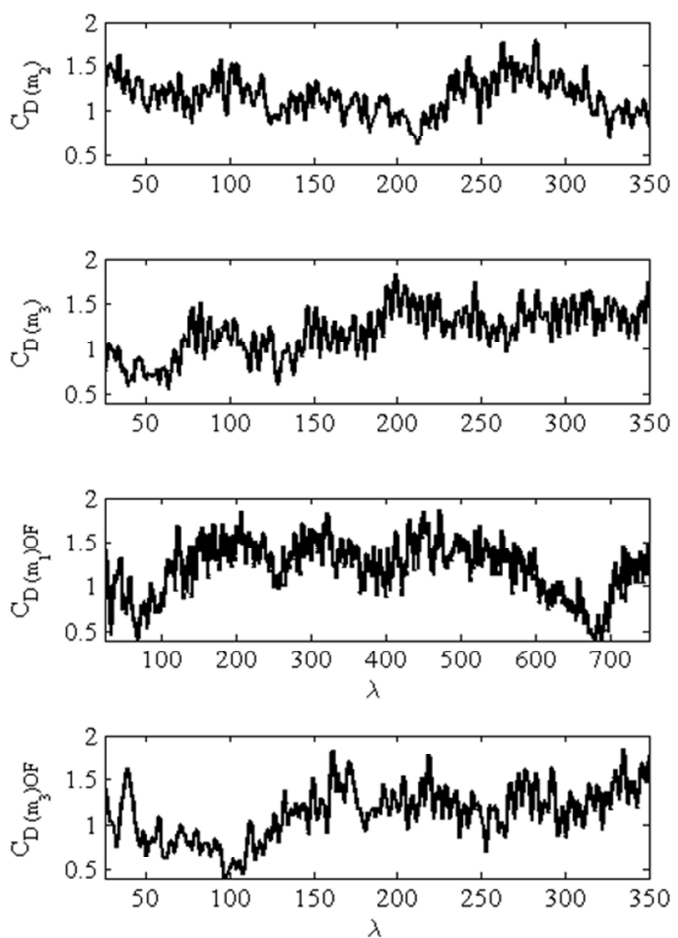

Fig. 3. Drag coefficient as a function of time obtained with mesh (a) $m_{1}$, (b) $m_{2}$, and (c) $m_{3}$ by using FLUENT and obtained with (d) $\mathrm{m}_{1}$ and (e) $\mathrm{m}_{3}$ by using OpenFOAM.

232 predicted by FLUENT and OpenFOAM are listed in Table 3. Time steps and mesh density used

233 are also listed in Table 3. Strouhal number is determined by performing fast Fourier Transform 
234 to the time signature of the lift coefficient of P1 and P2. Strouhal number predicted using $\mathrm{m}_{1}, \mathrm{~m}_{2}$

235 and $m_{3}$ are around 0.096 ; implying that time signature of hydrodynamic loadings is not sensitive

236 to the mesh density. Strouhal number is defined by $S t=f D / U_{\infty}$, where $f$ is the frequency of

237 fluctuating lift coefficient, $C_{L}$. Lift coefficient is defined by $C_{L}=F_{L} /\left(\frac{1}{2} \rho U_{\infty}^{2} A\right)$, where $F_{L}$ is the

238 lift force exerted by fluid on the prism. The lift force acting on finite prisms has more

239 complicated dynamics when compared to that on infinite prisms. Smaller amplitude fluctuations

240 are observed in the time signature of drag coefficient for P1 compared to that for P2. That is

241 attributed to the turbulent nature of induced wake flows as oncoming flows for P2 (see Fig. 3).

242 Time averaged value of $C_{D}$ for $\mathrm{m}_{1}, \mathrm{~m}_{2}$ and $\mathrm{m}_{3}$ are similar, as listed in Table 3. Drag coefficient

243 for $m_{1}$ are also very close at $\Delta \lambda=0.004$ and at $\Delta \lambda=0.012$. Due to the irregular dynamics rms

244 values listed in Table 3 are relatively large, especially for P2 prism. It is demonstrated here that

245 grid $\mathrm{m}_{1}$ and time step of 0.004 are sufficient to ensure spatial and temporal convergence. Results

246 presented in the present paper are obtained using mesh $\mathrm{m}_{1}$ and $\Delta \lambda=0.004$. Stronger claim for

247 spatial and temporal convergence could be made by refining the mesh further and using smaller

248 time step. The level of computation and available computational resources make that to be a

249 challenging task.

250 Table 3. Mean drag coefficient and rms value of P1 and P2.

\begin{tabular}{|c|c|c|c|cc|cc|}
\hline \multirow{2}{*}{$\begin{array}{c}\text { Case } \\
\text { No. }\end{array}$} & \multirow{2}{*}{ Mesh Size } & \multirow{2}{*}{ Solver } & Time step & \multicolumn{3}{|c|}{ P1 } & \multicolumn{2}{|c|}{ P2 } \\
\cline { 4 - 7 } & & $\Delta \lambda$ & $\overline{\boldsymbol{C}}_{\boldsymbol{D}}$ & $\boldsymbol{C}_{\boldsymbol{D r m s}}$ & $\overline{\boldsymbol{C}}_{\boldsymbol{D}}$ & $\boldsymbol{C}_{\boldsymbol{D r m} \boldsymbol{s}}$ \\
\hline $\mathbf{5}$ & $\mathbf{m}_{\mathbf{1}}$ & FLUENT & 0.004 & 1.46 & 0.06 & 1.12 & 0.30 \\
$\mathbf{5}$ & $\mathbf{m}_{\mathbf{1}}$ & OpenFOAM & 0.01 & 1.54 & 0.09 & 1.23 & 0.30 \\
$\mathbf{5}$ & $\mathbf{m}_{\mathbf{2}}$ & FLUENT & 0.004 & 1.48 & 0.04 & 1.13 & 0.20 \\
$\mathbf{5}$ & $\mathbf{m}_{\mathbf{3}}$ & FLUENT & 0.004 & 1.48 & 0.04 & 1.26 & 0.26 \\
$\mathbf{5}$ & $\mathbf{m}_{\mathbf{1}}$ & FLUENT & 0.012 & 1.49 & 0.04 & 1.22 & 0.21 \\
$\mathbf{5}$ & $\mathbf{m}_{\mathbf{3}}$ & OpenFOAM & 0.0025 & 1.45 & 0.03 & 1.17 & 0.27 \\
\hline $\mathbf{3}$ & $\mathbf{m}_{\mathbf{1}}$ & FLUUNT & 0.004 & 1.51 & 0.04 & - & - \\
$\mathbf{3}$ & $\mathbf{m}_{\mathbf{1}}$ & OpenFOAM & 0.007 & 1.47 & 0.04 & - & - \\
\hline
\end{tabular}


Table 4 lists the maximum value of Courant number and $y^{+}$values of $\mathrm{P} 1$ and $\mathrm{P} 2$ for test

252 cases. Courant number, $C=u \Delta t / \Delta x$, is a non-dimensional stability indicator that shows if

253 solution satisfies the Courant-Freidrichs-Lewy (CFL) condition of $C \leq 1$. This condition ensures

254 the numerical stability of simulations. Courant number in all test cases is bounded by 1.67 and

255 the maximum value is only observed at few cells in the vicinity of prisms. $y^{+}$value of P1 and P2

256 for $\mathrm{m}_{1}, \mathrm{~m}_{2}$, and $\mathrm{m}_{3}$ in case 5 are ranging from 8 to 38 . Smaller value of $y^{+}$indicates a better

257 quality of mesh. Mean drag coefficient of both prisms predicted using $\mathrm{m}_{1}$ and $\mathrm{m}_{3}$ mesh density

258 are very similar. Even though $\mathrm{m}_{3}$ provides a better quality of mesh, grid $\mathrm{m}_{1}$ is selected to conduct

259 simulations here to reduce computational cost.

260 Table 4. Maximum value of Courant number and $y^{+}$of $\mathrm{P} 1$ and $\mathrm{P} 2$.

\begin{tabular}{|c|c|c|c|c|c|c|}
\hline \multirow{2}{*}{$\begin{array}{l}\text { Case } \\
\text { No. }\end{array}$} & \multirow{2}{*}{ Mesh Size } & \multirow{2}{*}{ Solver } & \multirow{2}{*}{$\begin{array}{c}\text { Time step } \\
\Delta \lambda\end{array}$} & \multirow{2}{*}{$C_{\max }$} & P1 & $\mathbf{P 2}$ \\
\hline & & & & & $y^{+}$ & $y^{+}$ \\
\hline 5 & $\mathbf{m}_{1}$ & FLUENT & 0.004 & 0.34 & 30.03 & 33.65 \\
\hline 5 & $\mathbf{m}_{1}$ & OpenFOAM & 0.01 & 0.99 & 22.91 & 28.02 \\
\hline 5 & $\mathbf{m}_{2}$ & FLUENT & 0.004 & 0.79 & 10.09 & 26.07 \\
\hline 5 & $\mathbf{m}_{3}$ & FLUENT & 0.004 & 1.67 & 8.47 & 8.04 \\
\hline 5 & $\mathbf{m}_{3}$ & OpenFOAM & 0.0025 & 0.99 & 7.98 & 7.63 \\
\hline 5 & $\mathbf{m}_{1}$ & FLUENT & 0.012 & 1.11 & 31.95 & 38.01 \\
\hline 3 & $\mathbf{m}_{1}$ & FLUENT & 0.004 & 0.57 & 10.63 & - \\
\hline 3 & $\mathbf{m}_{1}$ & OpenFOAM & 0.007 & 0.99 & 8.97 & - \\
\hline
\end{tabular}

262 case 3 and case 5 are compared. Isosurfaces of vorticity, contours of vorticity and velocity are

263 depicted in Fig. 4 for flows past two finite tandem prisms (case 5). Isosurfaces of vorticity are

264 presented at the Q-criterion of 0.01 . The Q-criterion is the second invariant of the velocity

265 gradient tensor that describe the vortex core regions (see Ref [34] for detailed description). The

266 Q-criterion is calculated by $\mathrm{Q}=-0.5\left(\|S\|^{2}-\|\Omega\|^{2}\right)$, where $S$ and $\Omega$ are the strain and the

267 rotation tensor, respectively. Isosurfaces of the Q-criterion is colored with the normalized 
268 vorticity magnitude, $\omega D / U_{\infty}$, where $\omega$ is the vorticity magnitude. Images on the left column

269 denote flow patterns predicted by FLUENT solver while images on the right column denote flow

270 field predicted by OpenFOAM solver. Flow characteristics obtained by both solvers are very

271 similar. Similar results are obtained for flow past a single prism (images not shown here). The

272 time signature in drag coefficient predicted by both solver is also very similar, as shown in Fig.

273 3. Drag coefficients predicted by FLUENT and OpenFOAM differ less than 6\% for P1 and less

274 than $9 \%$ for P2 for case 5. The difference in the drag coefficient is less than $3 \%$ for case 3 . Flow

275 field and hydrodynamic loading predicted by each solver is nearly the same, validating the 276 numerical methods employed here. 


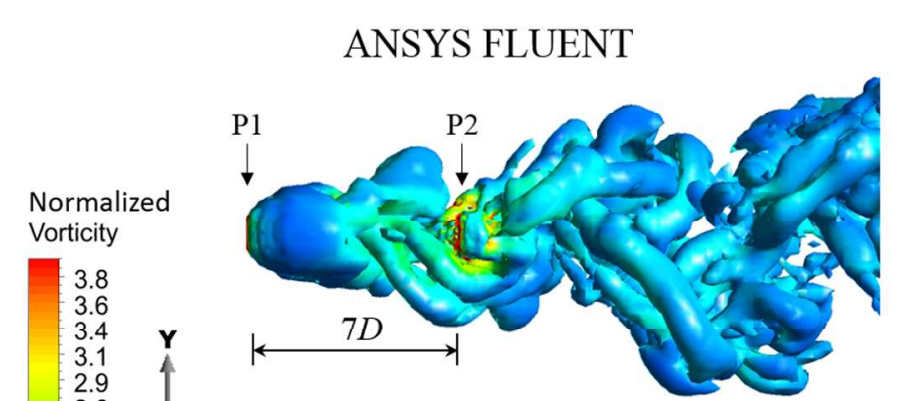

(a)

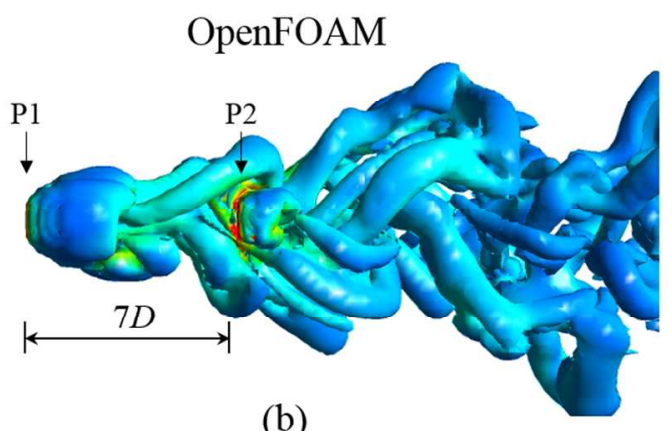

(b)

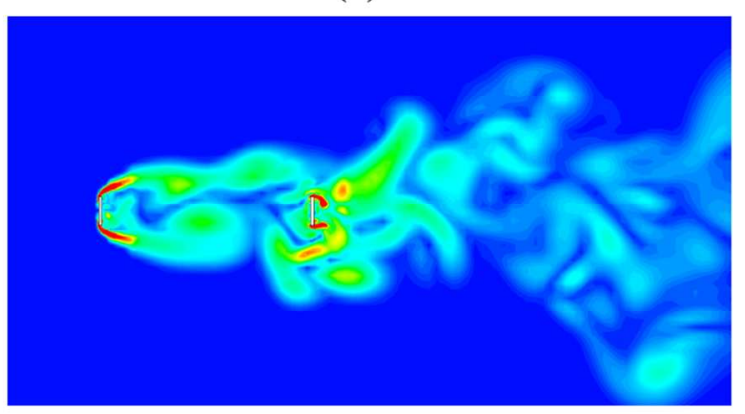

(d)

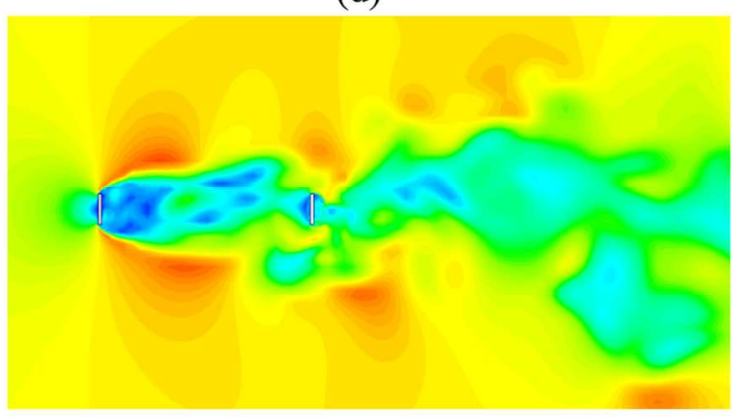

(e)

Normalized

Velocity

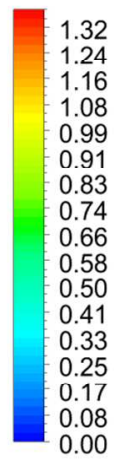

278

279

280

281

282

283

284

285

286

Fig. 4. (a,b) Isosurface of vorticity at $\mathrm{Q}=0.01,(\mathrm{c}, \mathrm{d})$ contours of vorticity, and $(\mathrm{e}, \mathrm{f})$ contours of velocity acquired at $y=0$ plane for case 5 . Results are obtained by using (a,c,e) ANSYS FLUENT and by using $(b, d, f)$ OpenFOAM solver.

\section{Validation study}

A simulation is conducted for flows past two tandem square cross-sectioned prisms in order to validate mathematical models and numerical methods employed. Results are compared against experimental results reported by Liu and Chen [10]. The schematic of the computational domain used in the validation study is depicted in Fig. 5(a) with the side view shown in Fig. 5(b). 
287 The upstream and downstream prisms are labeled as S1 and S2, respectively. The origin of the 288 computational domain is located at the center of S1. The height of cylinders is defined as $h$, 289 where $h$ is $0.2 \mathrm{~m}$. The spacing between cylinders from center to center is $4.25 h$. Inlet is located at $29018 \mathrm{~h}$ upstream of origin and the outlet is located at $37 \mathrm{~h}$ downstream of the origin. The height of 291 the computational domain is $15.25 h$ and the length in the span-wise direction is $10 h$. Reynolds 292 number is set to 2700 .
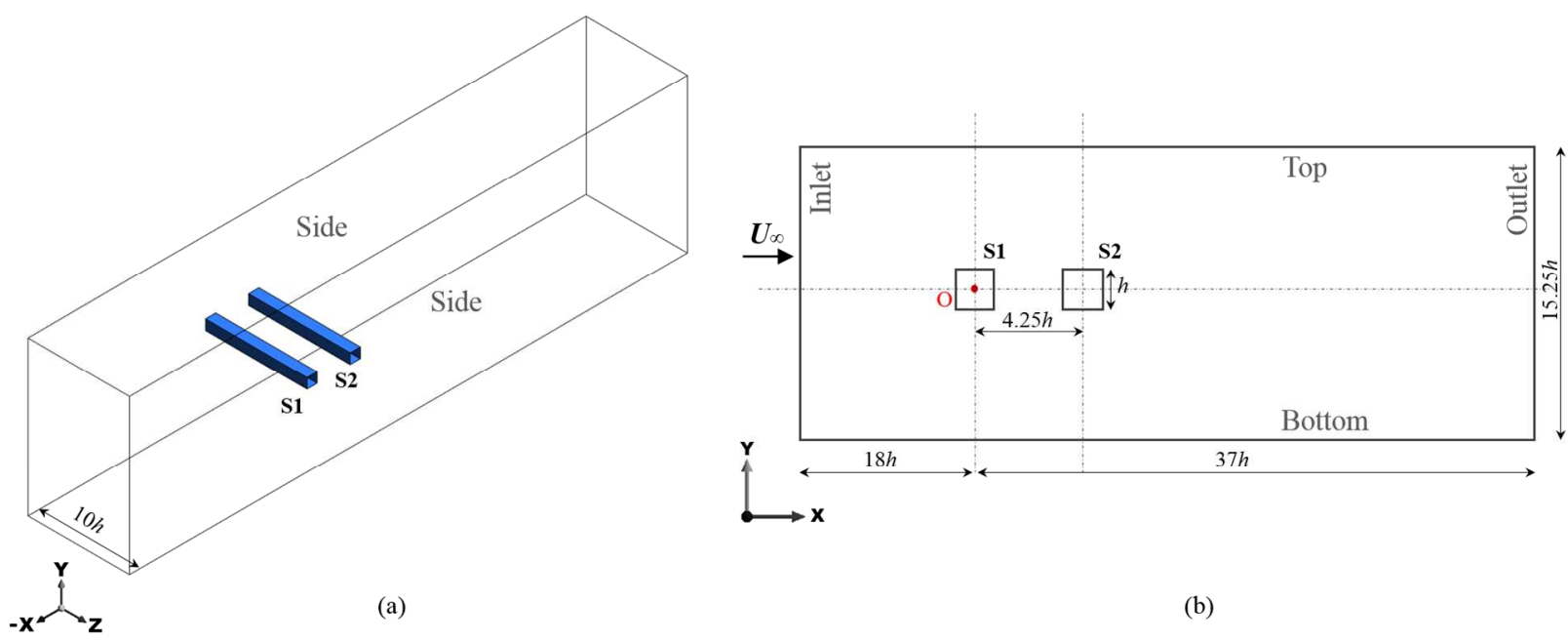

(b)

295 Fig. 5. Schematic diagram of flows past two tandem square cross-sectioned prisms in (a) a three296 dimensional view (b) a side view.

297 Drag and lift coefficients as a function of time and the pressure coefficient along the 298 surface of the prisms are depicted in Fig. 6. The hysteresis phenomenon induced by flow 299 transition is observed at this spacing of prisms, as reported by Liu and Cheng [10]. 300 Hydrodynamic loadings on prisms changes drastically as flow transition occurs. Flow 301 characteristics referred to as Mode I and Mode II are observed at different stages. Mode I is only 302 observed at the early stage of the flow while Mode II becomes persistent after the onset of flow 
303 transition. The vorticity contours acquired at $y=0$ plane demonstrate both flow patterns 304 associated with the Mode I and the Mode II, as depicted in Fig. 7. Similar to the observations, 305 flow patterns associated with the Mode I occurs at the earlier stage then the flow pattern switch 306 to Mode II following the flow transition. Flow patterns predicted by the present study match 307 qualitatively with those reported in Ref [10] for each mode. Drag coefficient of S1 prism for the 308 flow Mode I is obtained as 1.67 while it is 2.07 for the flow Mode II. Drag coefficient of S2 309 prism for the flow Mode I and II are -0.03 and 0.8 , respectively. These values match well with 310 those documented in Ref [10]. Drag coefficient values reported in Liu and Cheng [10] for S1 311 prism is around 1.6 for the flow Mode I and 1.9 2.1 for the flow Mode II. $C_{D}$ of S2 is around 3120.4 for the flow Mode I and 0.7 for the flow Mode II. Slight deviations in drag coefficient for S2 313 prism can be attributed to the fact that finer mesh might be needed to determine complex 314 unsteady wake flow structures near S2 prism, see Fig. 7. Lift coefficients of both prisms 315 transition to larger amplitude fluctuations as flow transition from the Mode I to the Mode II. The pressure coefficient, $C_{p}$, is defined by $C_{p}=\left(p-p_{\infty}\right) /\left(\frac{1}{2} \rho U_{\infty}^{2}\right)$, where $p_{\infty}$ is zero

317 gauge pressure. The pressure is significantly greater along the front face of the prism S1 and it 318 decreases rapidly to a lower value at the side and back faces for both flow Mode I and II. The 319 distribution of mean pressure coefficient predicted here along the surfaces of S1 and S2 are in 320 good agreement with experimental results reported in Ref [10]. Overall, the hysteresis 321 phenomenon, hydrodynamic loadings on prisms, and flow structures observed by Liu and Cheng 322 [10] are predicted by the current study; validating mathematical models and numerical methods 323 employed. 

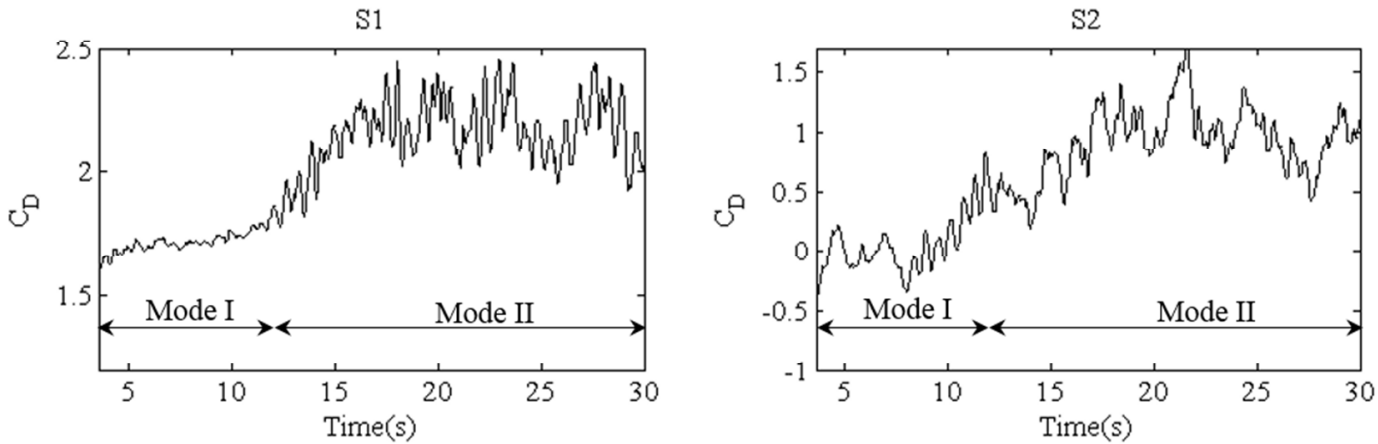

(a)

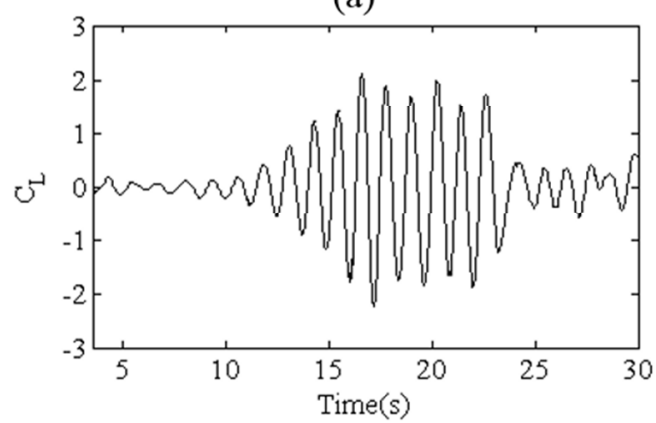

(b)

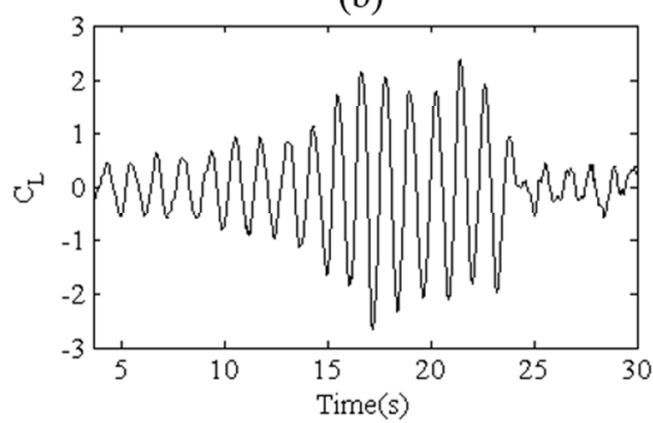

(c)

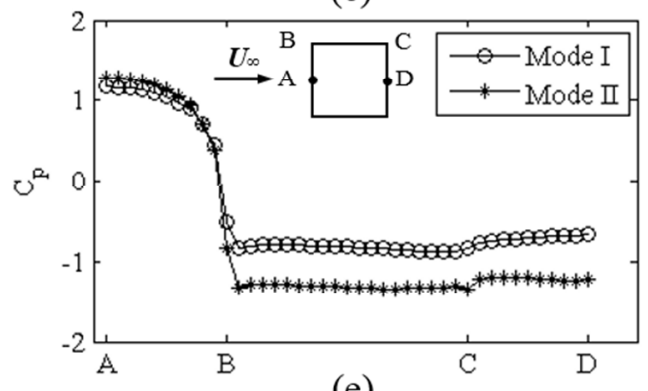

(e)

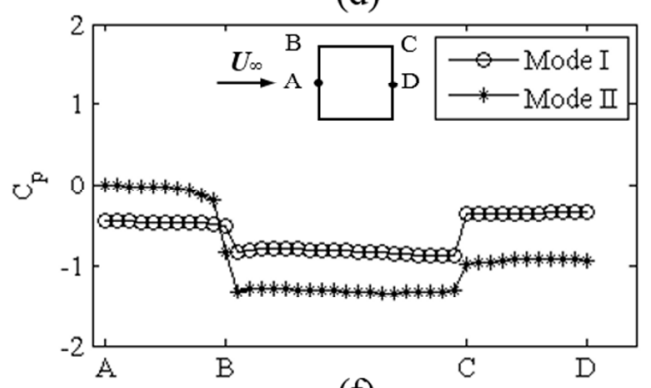

(f)

325 Fig. 6. (a,b) Drag coefficient and (c,d) lift coefficient as a function of time, and (e,f) distribution of pressure coefficient along surfaces of prisms. Force coefficients are calculated for (a,c,e) S1 prism and for $(b, d, e) S 2$ prism. 


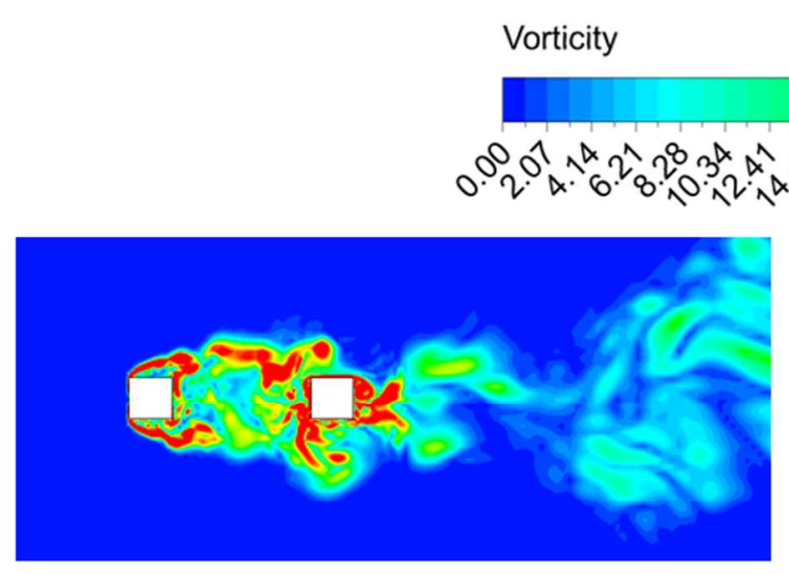

(a) Mode I $\left[\mathrm{s}^{\wedge}-1\right]$

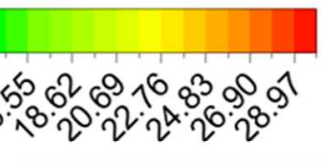

(b) Mode II

329

330

331

332

Fig. 7. Instantaneous vorticity contour acquired at $y=0$ plane for (a) flow Mode I at $6.2 \mathrm{sec}$ and for (b) flow Mode II at $16.6 \mathrm{sec}$.

\section{Results and Discussions}

\subsection{Flows past an infinite and a finite prism}

The drag coefficient of a finite prism is vastly different compared to that of an infinitely long prism, as documented by several investigators. It is also well-documented that drag coefficient of a finite prism strongly depend on the aspect ratio $L / D$. Transient simulations in three-dimensional geometry are conducted for flows past an infinitely long prism. Drag coefficient measured by Fage and Johansen [3] is 2.13 and predicted by Najjar and Vanka [4] and Tian et al. [5] is 2.26 and 2.30, respectively. The drag coefficient predicted by the current study is 2.22 , which agrees well with those documented by previous investigators. As a base case

342 (case 3), flows past a finite length prism with $L / D$ of 10 are simulated. The mean drag coefficient

343 is calculated to be 1.5 , which is $32 \%$ smaller compared to that of an infinitely long prism. The tip

344 vortices spawned from the prism's free ends make the flow highly three-dimensional. Fluid past 
345 free ends and wrap around the back of the prism. Such flow alters the pressure field and leads to

346 a reduction in drag coefficient. Isosurface of vorticity at $Q=0.01$, and the vorticity contour in

347 flows past a finite and an infinite prism are depicted in Fig. 8. Drag coefficient determined for

348 case 3 is used as a reference in flows past tandem prisms.

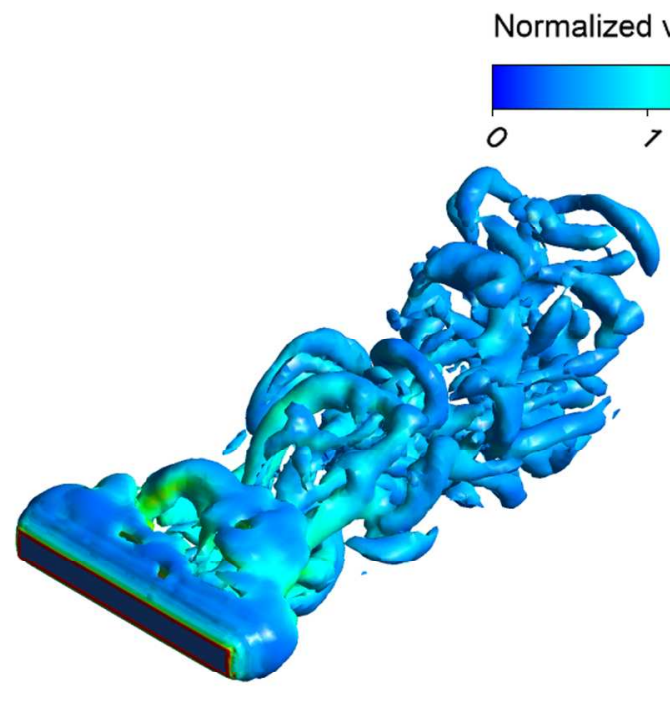

(a)

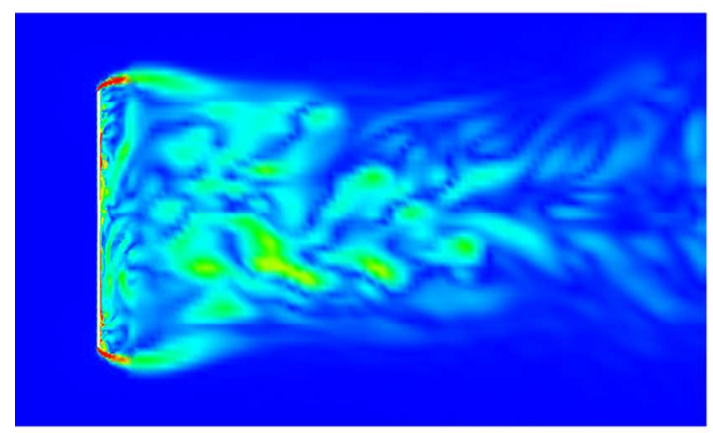

(c)
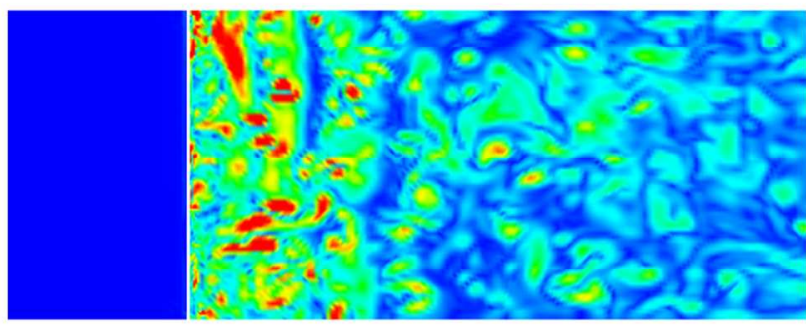

(d)

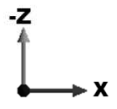

Fig. 8. $(\mathrm{a}, \mathrm{b})$ Isosurface of vorticity at $\mathrm{Q}=0.01$ and $(\mathrm{c}, \mathrm{d})$ contours of instantaneous vorticity at $y=$ 3520 plane. Images are acquired for $(a, c)$ a finite prism $(b, d)$ an infinite prism. 
355 in two-dimensional and three-dimensional geometries are conducted for the same $G / D$, and

356 results are shown in Fig. 9. The $x-y$ plane is used in simulations for a two-dimensional geometry.

357 Drag coefficient predicted in three-dimensional flows differ drastically compared to those 358 predicted in two-dimensional flows. Solid symbols denote drag coefficient for P1 while hollow 359 symbols denote drag coefficient for P2. Circles designate drag coefficients predicted by two360 dimensional transient LES simulations and squares designate drag coefficients predicted by 361 three-dimensional simulations. It is documented that two-dimensional flow models over predict 362 drag coefficient $[1,4]$. The drag coefficient of downstream prism, P2, is lower than that of P1.

363 Intense turbulent wake flows induced by P1 influence the drag coefficient of P2 strongly for low 364 values of $G / D$. As $G / D$ is increased the influence of wake decreases and drag coefficient of 365 downstream prism increases. Drag coefficient of both prisms predicted by three-dimensional 366 simulations become nearly the same for $G / D$ of 10 . On the other hand, drag coefficient predicted 367 by two-dimensional simulations for downstream prism rebound very little even for $G / D=20$, as 368 depicted in Fig. 9. It is clear that there is a flow transition occurring as $G / D$ is varied. The critical 369 value of $G / D$ for the onset of flow transition is around 7. The flow transition manifests itself by 370 rapid rebound in drag coefficient of the downstream prism P2. Obviously, two-dimensional flow 371 modeling fails to capture such flow transitions or it predicts the flow transition at values of $G / D$ 372 much greater than 20. Characteristics of flow transition can be identified better by examining 373 flow structures near prisms. 


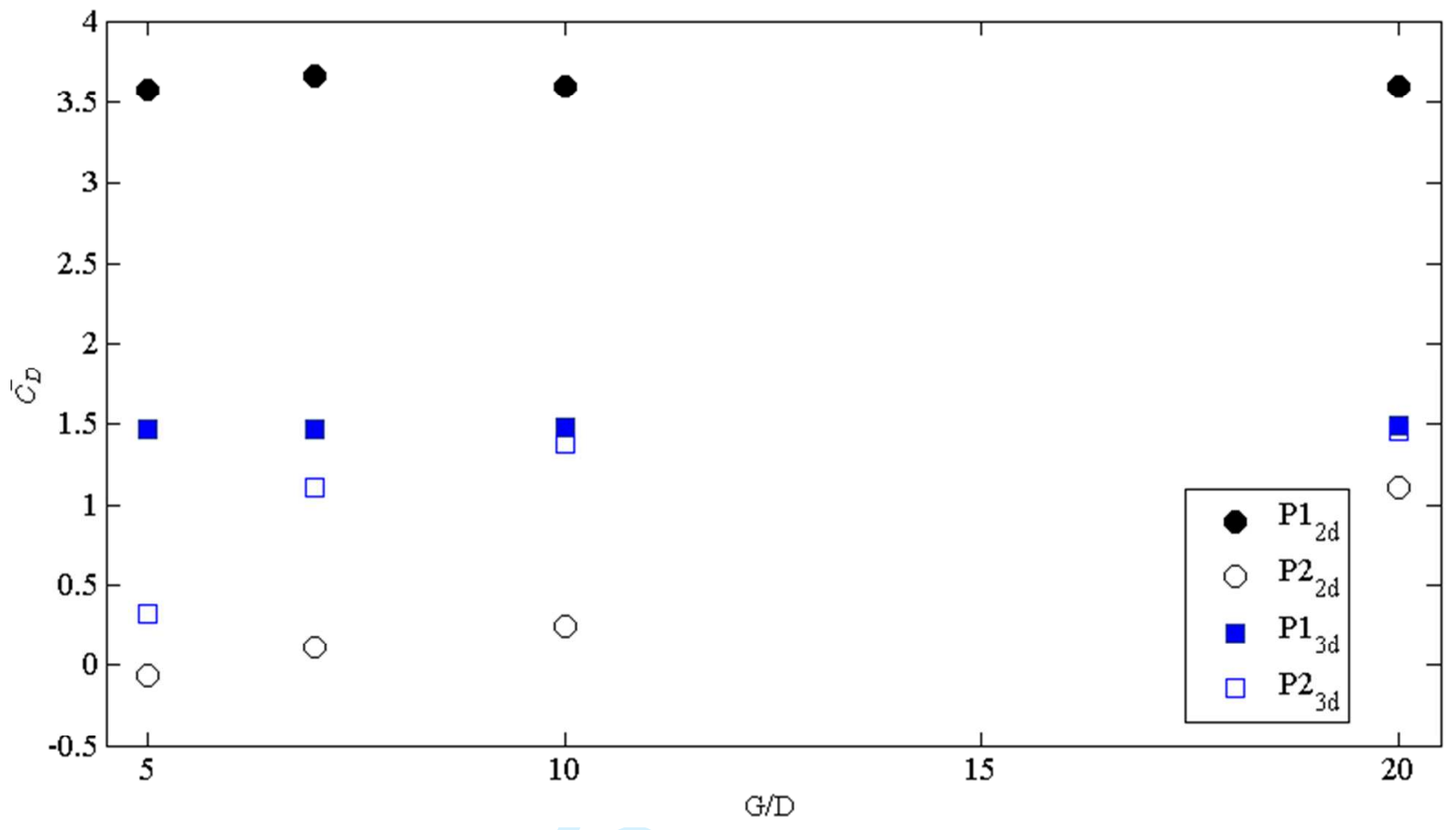

375

Fig. 9. Mean drag coefficient of P1 and P2 predicted by two- and three-dimensional simulations for various values of $G / D$.

Isosurface of vorticity, based on Q-criterion, and contours of vorticity in flows past two tandem prisms with different spacing are depicted in Fig. 10. Contours of magnitude of vorticity are depicted at $y=0$ plane for $G / D=5,7,10$ and 20. Streamlines originating from upstream of P1 are superimposed on images to better understand flow structures near prisms. Black lines in Fig. 10 denote streamlines. Streamlines originating from the mid-plane $(y=0)$ does not penetrate the wake of prisms. It should be noted that flows studied here are three-dimensional and streamlines from other planes will fill the wake region. Flow characteristics near two tandem

384 prisms are strongly influenced by the spacing between prisms, as also reported by Sohankar [12].

385 When two prisms are apart by $5 D$, the downstream prism, $\mathrm{P} 2$, is shielded by the shear layers 386 induced by the upstream prism, P1. The intensity of vorticity field downstream and upstream of 387 P2 is similar, hence it is expected that the pressure difference between the upstream and 
388 downstream face of P2 is smaller. This translates to radical drop in drag coefficient of this prism.

389 For $G / D=7$, shear layers formed from P1 reattach onto P2, upstream and downstream of P2

390 displays noticeable difference in flow patterns. As a result the drag coefficient of P2 rebounds, as

391 illustrated in Fig. 9. For $G / D=10$, both prisms start generating the Karman vortex streets

392 individually. Vortices generated from P1 still impinge on P2 periodically, as shown in Fig. 10(e)

393 and Fig. 10(f). The intensity of vortices impinging on P2 is decreased significantly for $G / D=20$.

394 The drag coefficient of both P1 and P2 becomes nearly the same for $G / D=20$ (see Fig. 9). 
Normalized vorticity

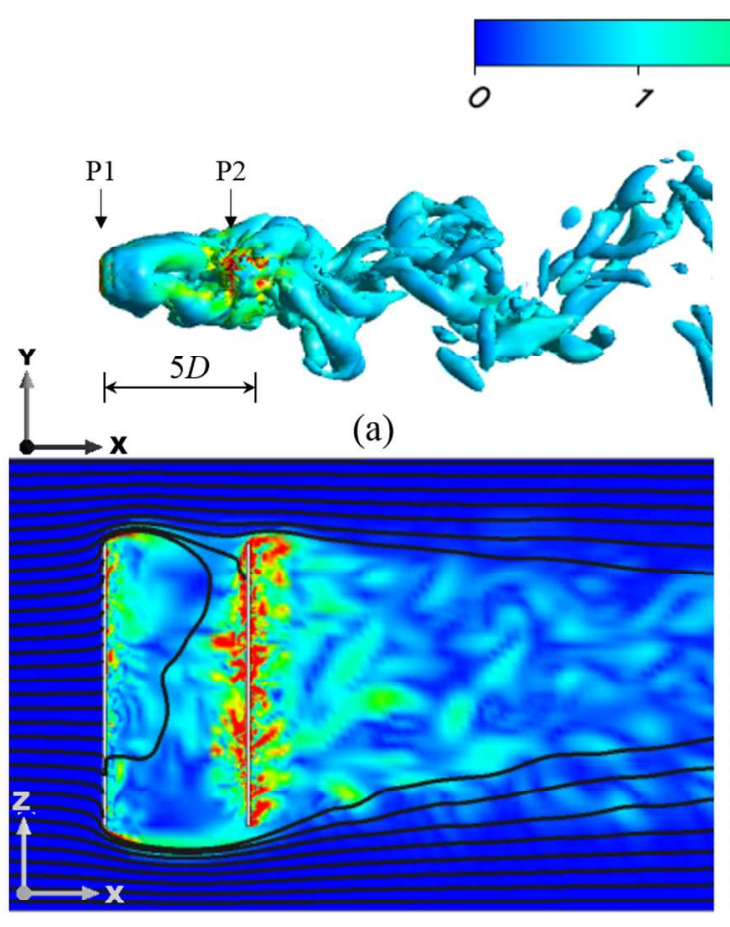

(b)
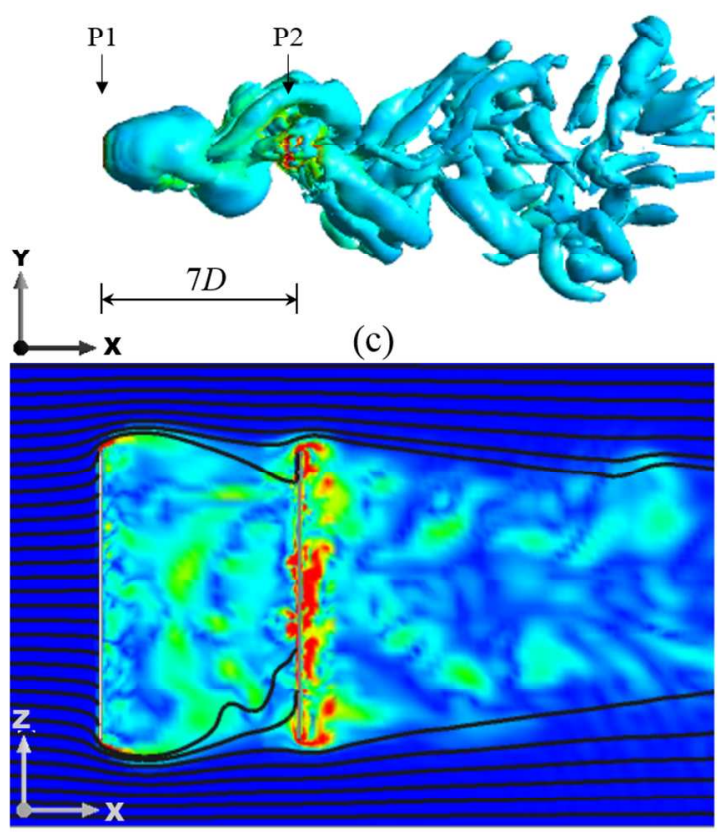

(d)

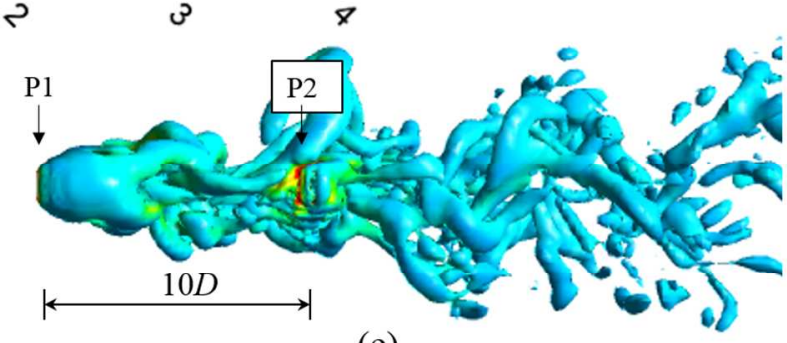

(e)

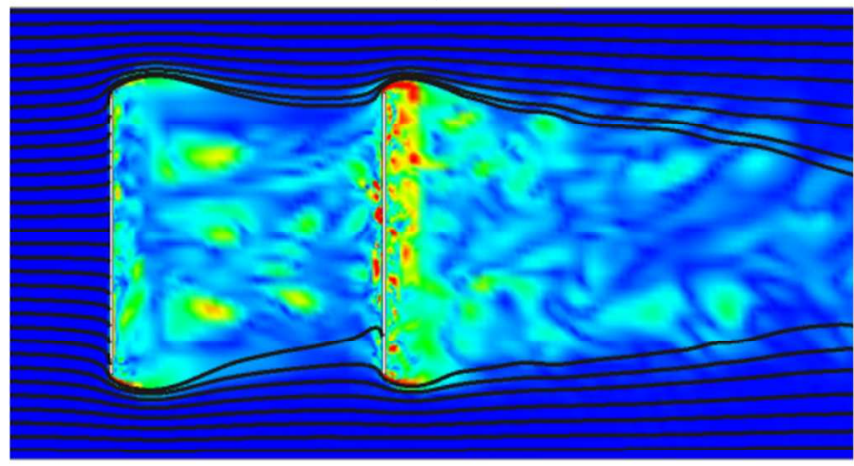

(f)

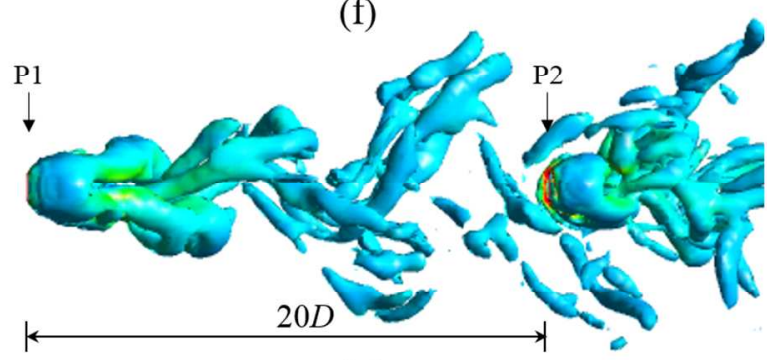

(g)

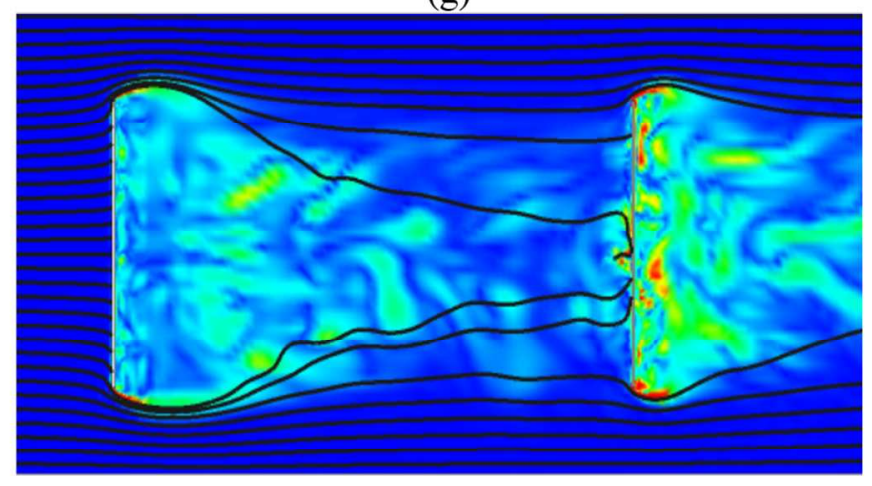

(h)

396 Fig. 10. (a,c,e,g) Isosurface of vorticity at $Q=0.01$ and $(b, d, f, h)$ contours of instantaneous 397 vorticity in flows past two tandem prisms for different values of $G / D$. Prisms are separated by $3985 D$ (case 4), $7 D$ (case 5), 10D (case 6), and 20D (case 7), as illustrated in the figure. 


\subsection{Flows past an yawed prism}

$400 \quad$ Flows past a single prism for different values of the yaw angle are simulated. Drag 401 coefficient for the yaw angle of $45^{\circ}$ (case 1 ), $60^{\circ}$ (case 2), and $90^{\circ}$ (case 3 ), is calculated to be $4021.08,1.42$, and 1.50, respectively. Isosurface and contours of instantaneous vorticity for these 403 three cases are depicted in Fig. 11. Contours are acquired at the $y=0$ plane. In cases 1 and 2 , 404 there is a stationary tip vortex attached to the leading edge of the prism, as shown in Fig. 11(a)405 Fig. 11(d) and Fig. 11(b)-Fig. 11(e). Flow patterns in the wake become less turbulent. Absence 406 of large and small eddies are obvious from images. As the yaw angle is decreased, the turbulent 407 intensity decreases and the region where turbulent eddies present shrinks. This well-documented 408 streamlining effect will result in decrease in the drag coefficient, as predicted in the present 409 study. 


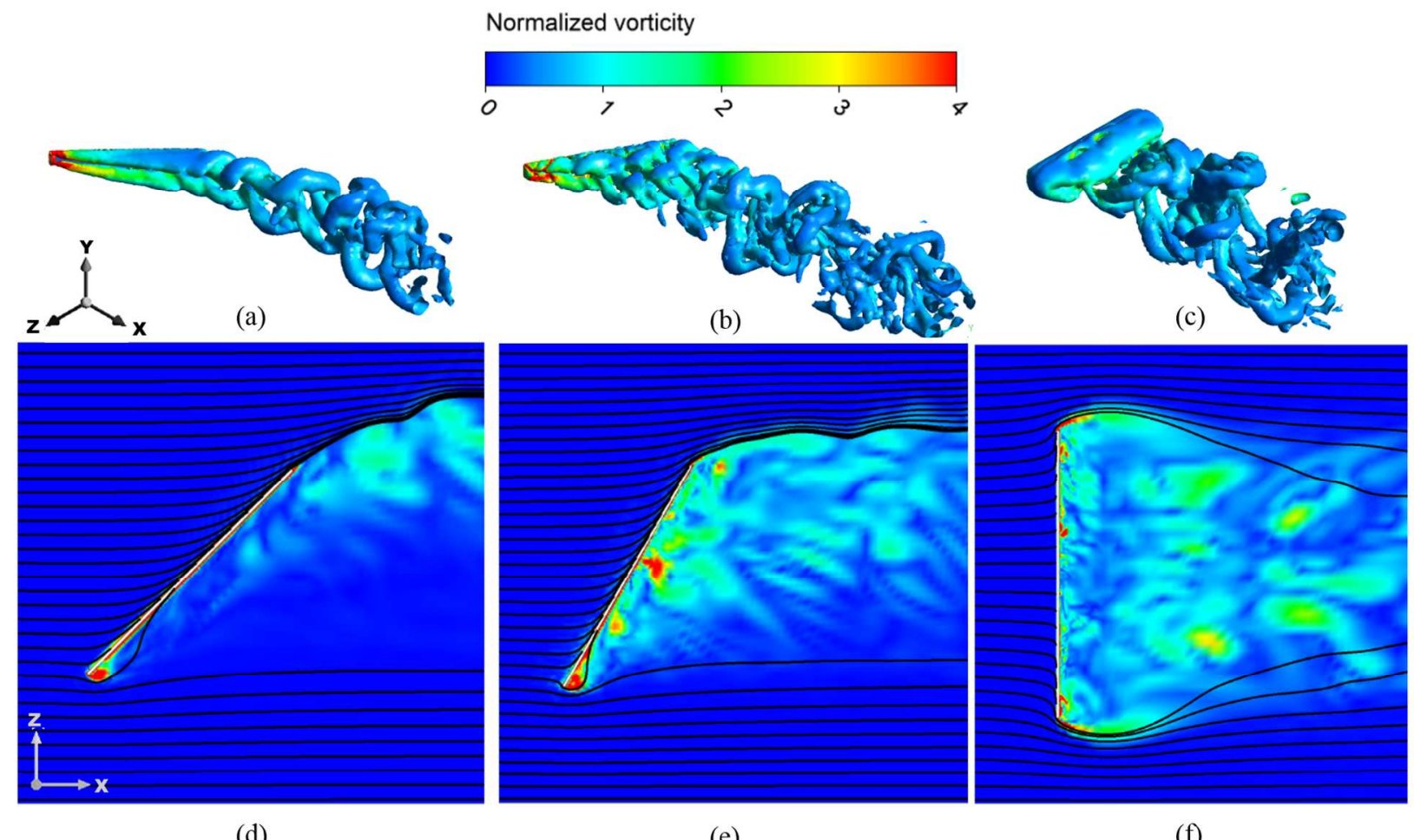

(d)

(e)

(f)

411 Fig. 11. $(a, b, c)$ Isosurface of vorticity at $Q=0.01$ and $(d, e, f)$ contours of instantaneous vorticity. 412 Images are acquired for the yaw angle of (a,d) $45^{\circ}$ (case 1), (b,e) $60^{\circ}$ (case 2), and (c,f) $90^{\circ}$ (case 413 3).

Flows past two yawed prisms in a staggered array are studied for a yaw angle of $45^{\circ}$ (case

4168 ) and $60^{\circ}$ (case 9). Simulations for both cases are conducted for $G / D$ of 10 . Drag coefficient of

417 prism $\mathrm{A} 1$ and $\mathrm{A} 2$ for case 8 is calculated to be 1.06 and 1.13, respectively. For case 9, drag

418 coefficient of prism A1 and A2 is 1.41 and 1.46. For each case, drag coefficient of downstream

419 prism is slightly higher than that of upstream prism. This slight increase in drag coefficient of

420 downstream prism can be ascribed to the observation that the fluid accelerating passing prism A1

421 is transported toward prism A2. Flow pattern for case 8 and case 9 are depicted in Fig. 12.

422 Stationary tip vortex is present in both prisms. Vortices shed from each prism roll up toward the 423 center, and are convected downstream, as shown in Fig. 12. The region where vortices are 
424 concentrated becomes smaller as the yaw angle is decreased. It is also noticed that as the yaw

425 angle is increased the shear layer near the centerline is formed. The wake of each prism is 426 separated by the shear layer.

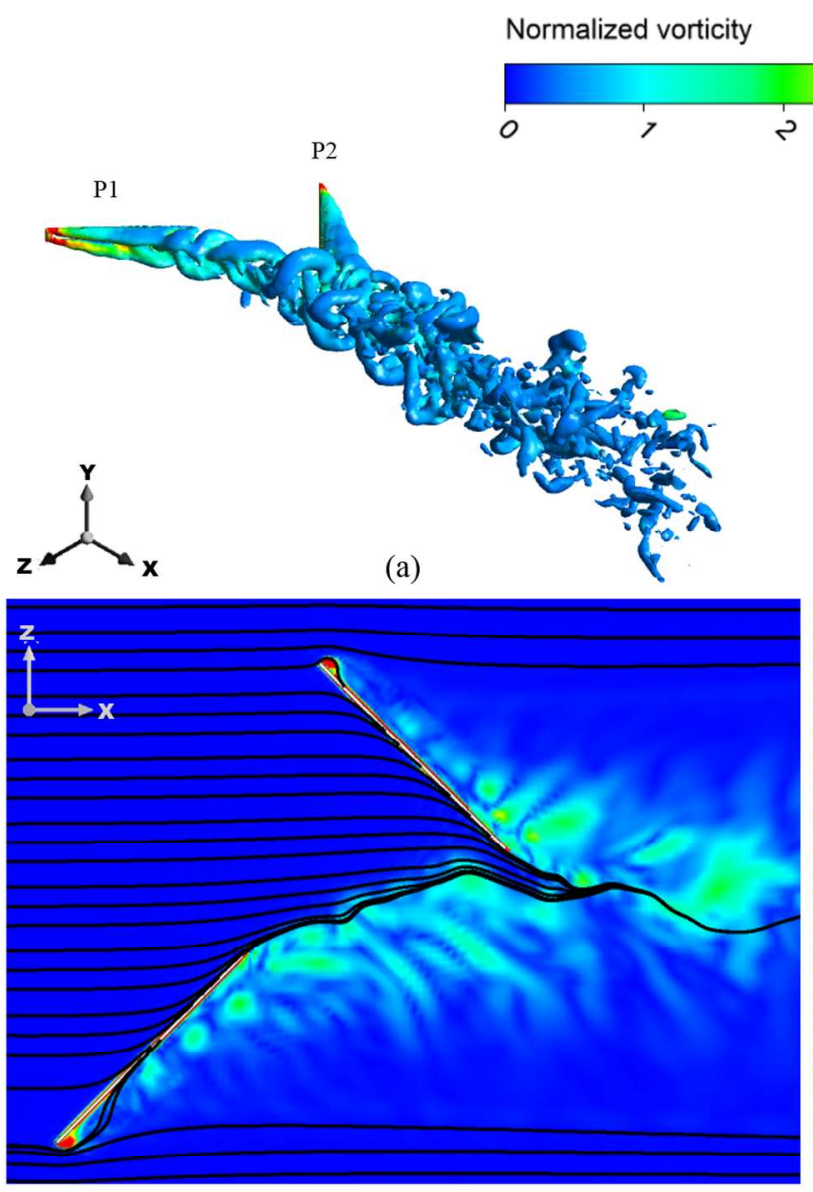

(c)
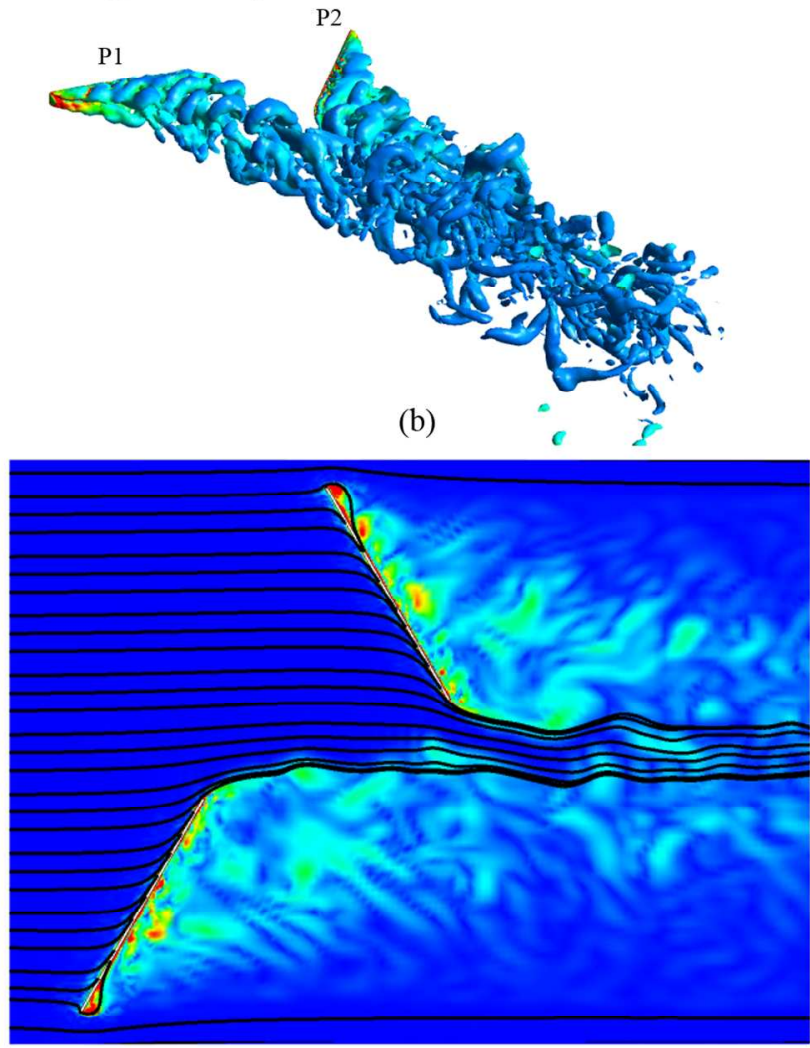

(d) 429 Fig. 12. (a,b) Isosurface of vorticity at $Q=0.01$ and $(c, d)$ contours of instantaneous vorticity for
430 two prisms in a staggered arrangement. Prisms have the yaw angle of $(a, c) 45^{\circ}($ case 8$)$ and $(b, d)$ $43160^{\circ}$ (case 9).

Simulations of three-dimensional flows past four yawed prisms are conducted with the

433 yaw angle of $45^{\circ}$ (case 10) and $60^{\circ}$ (case 12) in a staggered arrangement for $G / D$ of 10 . Drag 434 coefficient of each prism in cases 10 and 12 is calculated. Drag coefficient of A1, A2, A3 and A4 435 is 1.06, 1.13,1.09, and 1.09, respectively, for case 10. Drag coefficient of prisms for case 12 is 
$4361.38,1.46,1.32$, and 1.34, respectively, for A1, A2, A3 and A4. It is noted that drag coefficient

437 for A2 again is slightly larger than that of others for both cases. Drag coefficient of A3 and A4

438 are similar to that of A1; indicating that the turbulent wake flow patterns induced by upstream

439 prisms are hardly influencing the force exerted on these prisms. Flow images depict that vorticity

440 shed from upstream prisms still impinges on part of the downstream prisms, as shown in Fig. 13.

441 For yaw angle of $45^{\circ}$ and smaller, vortices impinge on a small region near trailing edge of 442 upstream prisms. Stationary tip vortex is attached to the leading edge of each prism in both cases,

443 as depicted in Fig. 13. As the number of prisms in staggered arrays is increased the drag 444 coefficient of downstream prisms remains the same. This implies that wake flow patterns are 445 repeated behind each prism. Drag coefficient of six yawed prisms in a staggered array (case 14) 446 are $1.06,1.12,1.10,1.10,1.12$, and 1.12, respectively, for A1 through A6. Prisms with spacing $447 G / D$ of 10 are situated at $45^{\circ}$ yaw angle against the oncoming flow. Flow patterns near prisms for 448 case 14 are depicted in Fig. 14. Downstream prisms are not experiencing highly turbulent wake 449 flows as oncoming flow toward them. That could be an important design consideration for 450 offshore and marine structures. Highly turbulent flows impinging on structures increase the risk 451 of failures in these systems. 


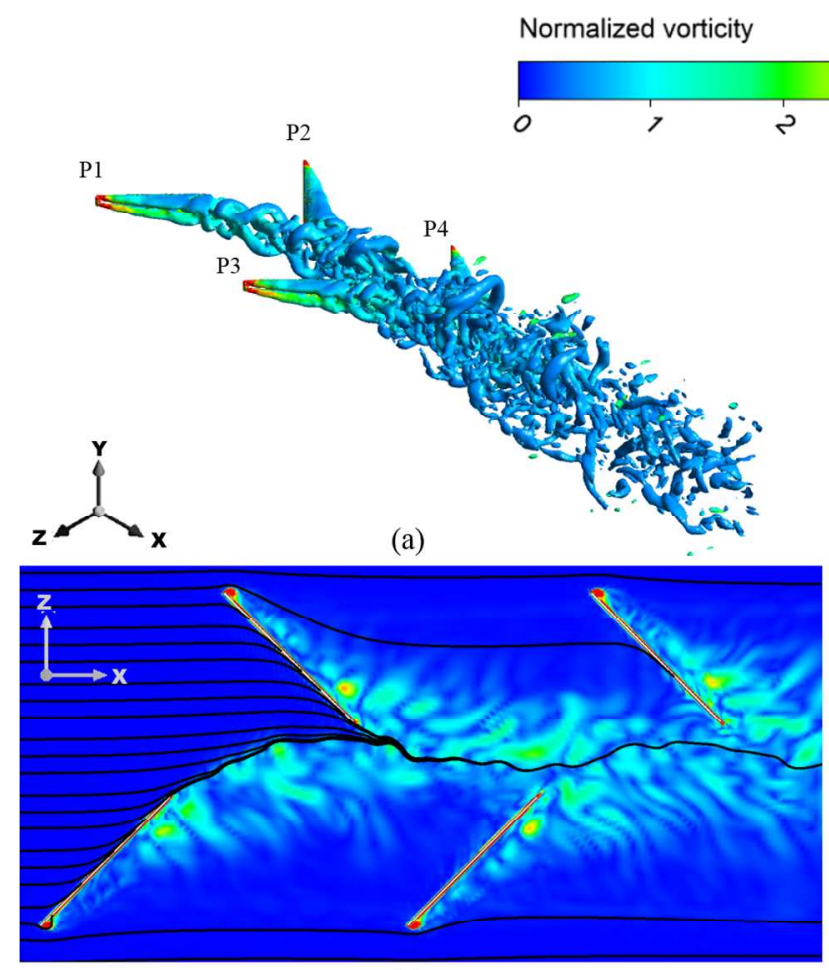

(c)

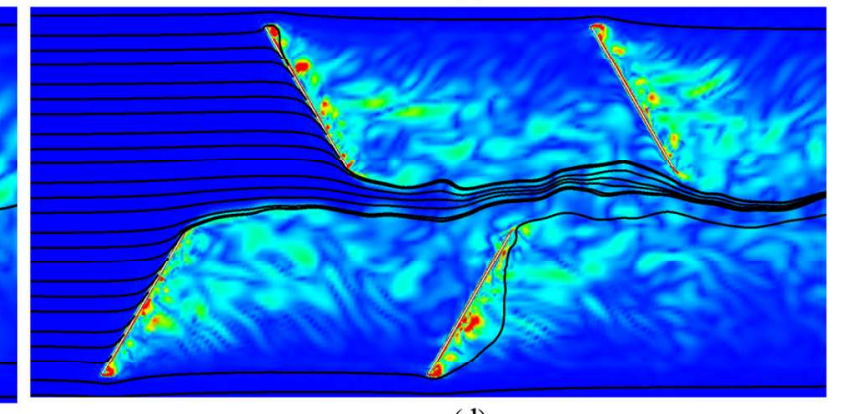

(d)

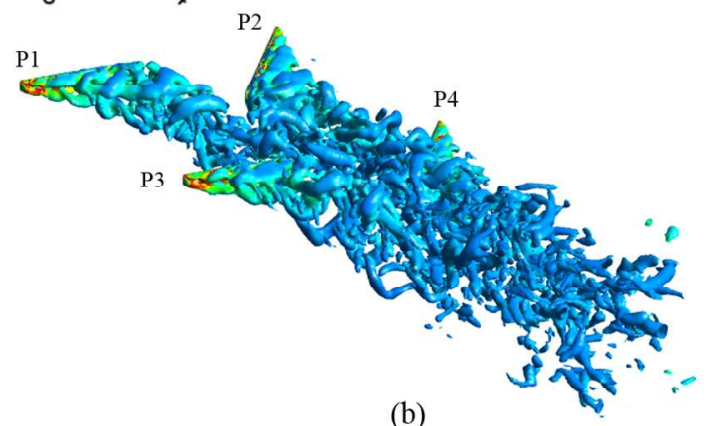

453 Fig. 13. (a,b) Isosurfaces of vorticity at $Q=0.01$ and $(c, d)$ contours of instantaneous vorticity for 454 four staggered prisms. Images are acquired for the yaw angle of (a,c) $45^{\circ}$ (case 10) and (b,d) $60^{\circ}$ 455 (case 12). 


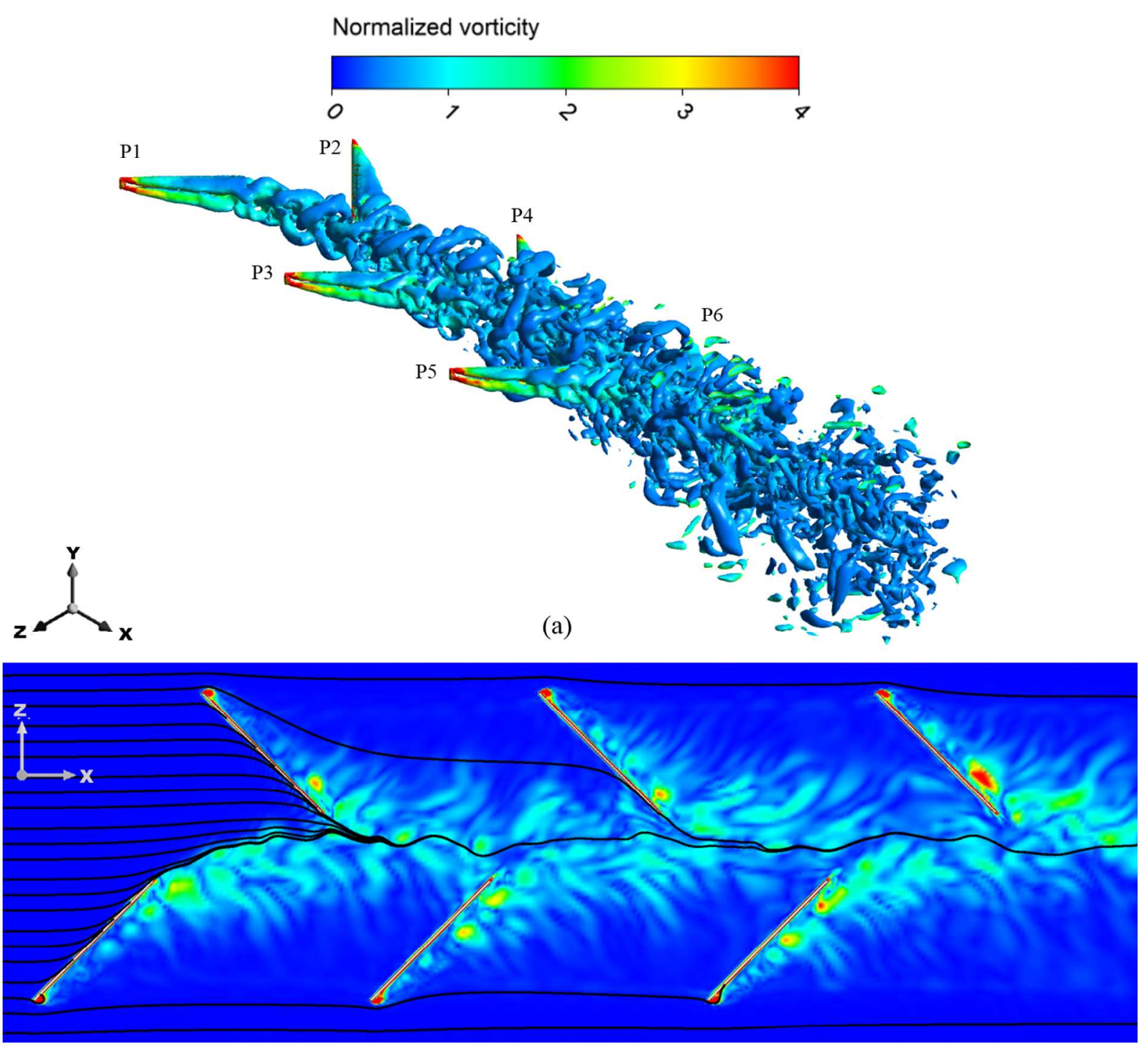

(b)

457 Fig. 14. (a) Isosurface of vorticity at $Q=0.01$ and (b) contours of instantaneous vorticity for six 458 staggered prisms with the yaw angle $45^{\circ}$ (case 14 ).

459 Fig. 15 illustrates flow patterns near four yawed prisms with yaw angle of $45^{\circ}$ and 460 spacing of $G / D=2.5$ and 5. Even though prisms are tightly spaced drag coefficient of 461 downstream prism is still $66 \%$ of the drag coefficient of upstream prisms for the case of $G / D=$ 462 2.5. Drag coefficient is calculated to be $1.04,1.08,0.66$, and 0.66 . It is clear that when prisms are 463 closely spaced, staggered arrays of yawed prisms will perform much better, as listed in Table 5. 
464 Drag coefficient of downstream and upstream prisms in a tandem and a staggered array are 465 compared in Table 5. For staggered yawed prisms drag coefficient of downstream prism is 466 slightly smaller for tight spacing. For perpendicular prisms in a tandem arrangement with 467 spacing of $G / D=5$ - not as tight as in case 11 - drag coefficient drops nearly $78 \%$ for the 468 downstream prism, as listed in Table 5 for case 4 . Tightly packed arrangement is desirable to be 469 used in energy harvesting devices for marine current applications. Staggered arrangements of 470 yawed prisms could be an integral part of design and optimization processes in these systems.

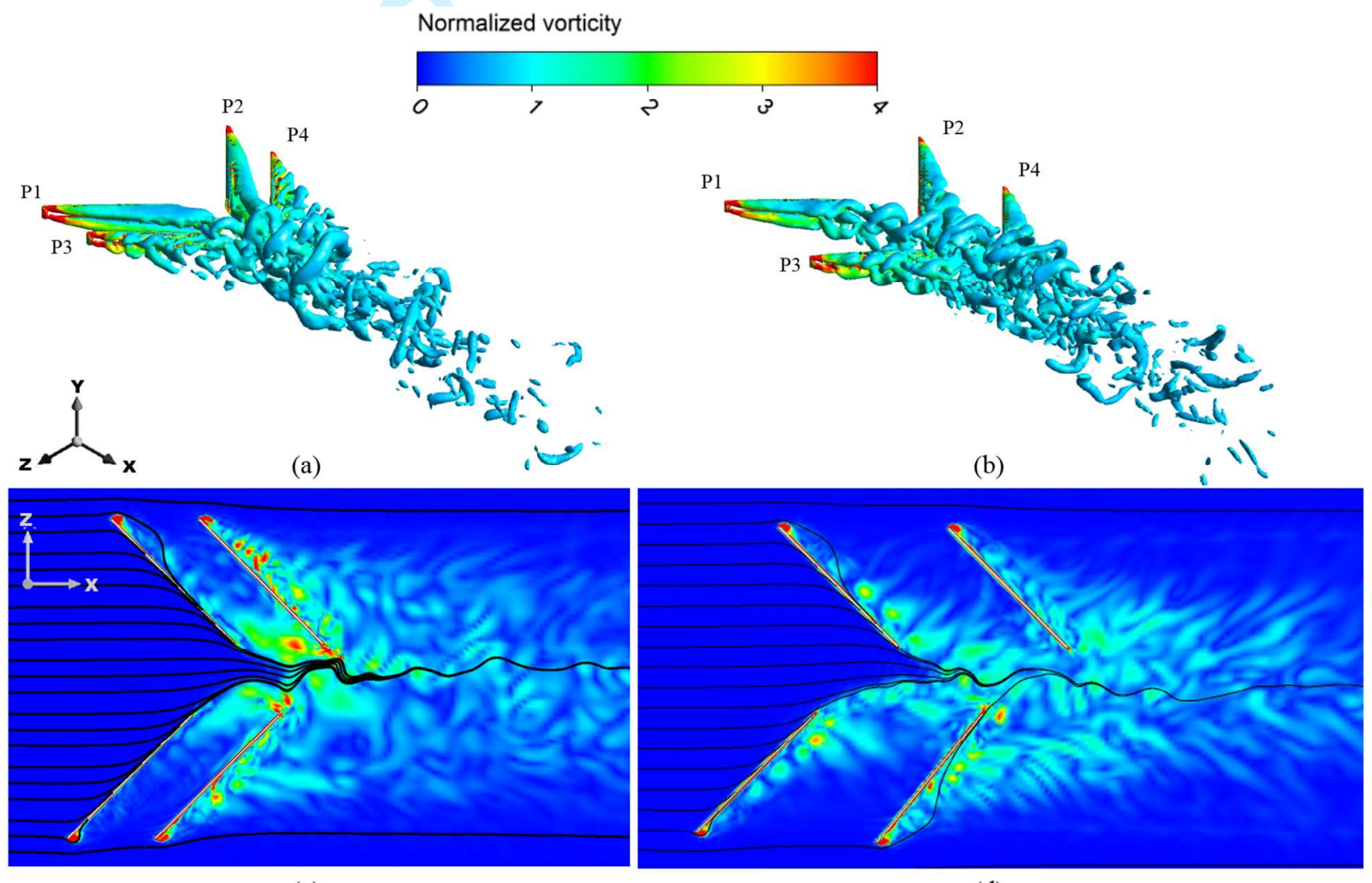

(c)

(d)

472 Fig. 15. (a,b) Isosurfaces of vorticity at $\mathrm{Q}=0.01$ and $(\mathrm{c}, \mathrm{d})$ contours of instantaneous vorticity for 473 four staggered prisms. Prisms are placed with the yaw angle of $45^{\circ}$ and the spacing of (a,c) $2.5 D$ 474 (case 11) and (b,d) 5D (case 13). 
475 Table 5. Average value of drag coefficient of upstream and downstream prisms in a tandem arrangement of perpendicular prisms and a staggered arrangement of yawed prisms.

\begin{tabular}{|c|ccc|cc|}
\hline \multirow{2}{*}{$G / D$} & \multicolumn{2}{|c|}{ Tandem (case 4-7) } & \multicolumn{2}{c|}{ Staggered (case 11, 13, 10) } \\
\cline { 2 - 5 } & $\overline{\boldsymbol{C}}_{\boldsymbol{D} \_ \text {P1 }}$ & $\overline{\boldsymbol{C}}_{\boldsymbol{D} \_\mathbf{P 2}}$ & $\overline{\boldsymbol{C}}_{\boldsymbol{D} \_\boldsymbol{A} \mathbf{2}}$ & $\overline{\boldsymbol{C}}_{\boldsymbol{D} \_\boldsymbol{A 4}}$ \\
\hline $\mathbf{2 . 5}$ & - & - & 1.08 & 0.66 \\
$\mathbf{5}$ & 1.47 & 0.32 & 1.11 & 0.92 \\
$\mathbf{7}$ & 1.47 & 1.11 & - & - \\
$\mathbf{1 0}$ & 1.48 & 1.38 & 1.13 & 1.09 \\
$\mathbf{2 0}$ & 1.49 & 1.46 & - & - \\
\hline
\end{tabular}

\section{Conclusions}

LES simulations are carried out to study three-dimensional flows past arrays of finite 479 yawed prisms at $R e=50,000$ by FLUENT and OpenFOAM solver. Wake flow patterns are 480 characterized for different values of spacing $G / D$ and yaw angle in tandem and staggered arrays.

481 Finite prisms of $L / D=10$ with two free ends are used in simulations. The average value of drag 482 coefficient for each prism in tandem and staggered arrays are calculated. Results predicted by 483 FLUENT and OpenFOAM solvers agree well. Mathematical models and the numerical method 484 employed in the current study are validated by comparing simulated results against experimental 485 results reported by Liu and Chen [10].

As expected, drag coefficient of finite prism situated perpendicular to the oncoming flow

487 is lower compared to that of infinitely long prism. This is attributed to the three-dimensional

488 wake flows induced by the presence of tip vortices at free ends of finite prisms. The effect of 489 spacing in tandem arrays predicted by three-dimensional simulations is vastly different compared

490 to that predicted by two-dimensional modeling. For $G / D=5$, three-dimensional flow modeling 491 predicts that average drag coefficient of downstream prism is about $22 \%$ percent of drag 492 coefficient of upstream prism while simulations in two-dimensional geometry predict average 493 drag of upstream prism is nearly zero. Three-dimensional flow model also predicts that drag 
494 coefficient of downstream prism rebound quickly as $G / D$ is increased to 7 and recover almost 495 fully as $G / D$ is increased to 10 . On the other hand, drag coefficient predicted by simulations in 496 two-dimensional geometry rebound very slowly. Drag coefficient of downstream prism for $G / D$ 497 of 20 is still about $30 \%$ of the drag coefficient of upstream prism predicted by two-dimensional 498 modeling. Flow transitions observed in wake flows are responsible for the influence of spacing 499 on drag coefficients. For $G / D \leq 7$, the shear layers induced by P1 wraps around or reattaches to $500 \mathrm{P} 2$ and influences oncoming flows of downstream prism. For $G / D=10$ and higher, both 501 upstream and downstream prisms generate Karman vortex street almost independently.

503 prisms oriented perpendicular to oncoming flows. Nevertheless, when yawed prisms placed in 504 staggered arrays outcome could be very favorable in terms of stability and power generation for 505 marine current energy harvesting applications. Yawed prisms situated downstream part of the 506 array could be outside of the turbulent wake flows induced by upstream prisms. As presented by 507 the present study even for very tightly spaced yawed prisms $(G / D=2.5)$ drag coefficient of 508 downstream prisms can still be $63 \%$ of the drag coefficient of upstream prisms. Tighter spacing 509 of prisms aids in compact design of energy harvesting devices for the same power generation.

510 This study shows that designing and optimizing offshore structures requires accurate spatial and

511 temporal characteristics of the flows near structures. Orientation, arrangement and spacing of 512 these systems are critical variables in design and optimization of energy harvesting modules. 


\section{Acknowledgment}

516 Authors are thankful to Cosan Daskiran for his insightful discussion about numerical methods

517 employed here.

518

519 References

$520 \quad$ 1. X. Tian, M. C. Ong, J. M. Yang, and D. Myrhaug, Ocean Eng. 58, 208 (2013).

521 doi:10.1016/j.oceaneng.2012.10.013.

522 2. C. W. Knisely, J. Fluids Struct 4, 371 (1990). doi:10.1016/0889-9746(90)90137-T.

523 3. A. Fage and F. C. Johansen, Proceedings of the Royal Society A: Mathematical, Physical

524 and Engineering Sciences 116, 170 (1927). doi:10.1098/rspa.1927.0130.

525 4. F. M. Najjar and S. P. Vanka, Int. J. Numer. Methods Fluids 21, 525 (1995).

526 doi:10.1002/fld.1650210702.

$527 \quad$ 5. X. L. Tian, M. C. Ong, J. M. Yang, and D. Myrhaug, J. Fluids Struct 49, 149 (2014).

528 doi:10.1016/j.jfluidstructs.2014.04.008.

529 6. H. Sakamoto, H. Hainu, and Y. Obata, J. Wind Eng. Ind. Aerod. 26, 85 (1987).

530 doi:10.1016/0167-6105(87)90037-7.

531 7. I.-H. Liu, J. Riglin, W. C. Schleicher, and A. Oztekin, Ocean Eng. 111, 323 (2016).

532 doi:10.1016/j.oceaneng.2015.11.009.

533 8. T. P. Lloyd and M. James, Appl. Ocean Res. 59, 676 (2016).

534 doi:10.1016/j.apor.2015.11.009.

535 9. S. M. Yeon, J. M. Yang, and F. Stern, Appl. Ocean Res. 59, 663 (2016).

536 doi:10.1016/j.apor.2015.11.013.

537 10. C. H. Liu and J. M. Chen, J. Wind Eng. Ind. Aerod. 90, 1019 (2002). doi:10.1016/S0167-

538 6105(02)00234-9.

539 11. S. C. Yen, K. C. San, and T. H. Chuang, Exp. Therm. Fluid Sci. 32, 927 (2008).

$540 \quad$ doi:10.1016/j.expthermflusci.2007.07.001.

541 12. A. Sohankar, Theor. Comp. Fluid Dyn. 28, 531 (2014). doi:10.1007/s00162-014-0329-2.

542 13. D. Sumner, J. Fluids Struct 26, 849 (2010). doi:10.1016/j.jfluidstructs.2010.07.001.

543 14. M. Zhao, L. Cheng, B. Teng, and D. F. Liang, Appl. Ocean Res. 27, 39 (2005).

544 doi:10.1016/j.apor.2004.10.002.

545 15. F. Auteri, M. Belan, C. Cassinelli, and G. Gibertini, J. Visualization 12, 307 (2009).

546 doi:10.1007/bf03181875. 
16. M. Shirakashi, A. Hasegawa, and S. Wakiya, Bulletin of JSME 29, 1124 (1986). doi:10.1299/jsme1958.29.1124.

17. D. Yeo and N. P. Jones, J. Wind Eng. Ind. Aerod. 96, 1947 (2008). doi:10.1016/j.jweia.2008.02.040.

18. M. Zhao, L. Cheng, and T. M. Zhou, J. Fluids Struct 25, 831 (2009). doi:10.1016/j.jfluidstructs.2009.02.004.

19. S. A. Jordan, Int. J. Heat Fluid Flow 31, 489 (2010). doi:10.1016/j.ijheatfluidflow.2010.04.009.

20. H. F. Wang, Y. Zhou, C. K. Chan, and K. S. Lam, Phys. Fluids 18, 065106 (2006). doi:10.1063/1.2212329.

21. J. F. McClean and D. Sumner, J. Fluids Eng. 136, 081206 (2014). doi:10.1115/1.4027138.

22. D. Sumner, J. Fluids Struct 43, 41 (2013). doi:10.1016/j.jfluidstructs.2013.08.007.

23. T. Kawamura, M. Hiwada, T. Hibino, I. Mabuchi, and M. Kumada, B Jsme 27, 2142 (1984). doi:10.1299/jsme1958.27.2142.

24. N. Rostamy, J. McClean, D. Sumner, D. Bergstrom, and J. Bugg, Proceedings of the 7th International Colloquium on Bluff Body Aerodynamics and Applications (BBAA7)Shanghai, China, 2012.

25. M. M. Zdravkovich, V. P. Brand, G. Mathew, and A. Weston, J. Fluid Mech. 203, 557 (1989). doi:10.1017/S002211208900159X.

26. O. Inoue and A. Sakuragi, Phys. Fluids 20, 033601 (2008). doi:10.1063/1.2844875.

27. H. F. Wang and Y. Zhou, J. Fluid Mech. 638, 453 (2009). doi:10.1017/s0022112009990693.

28. G. Hu, K. T. Tse, K. C. S. Kwok, and Y. Zhang, J. Wind Eng. Ind. Aerod. 146, 172 (2015). doi:10.1016/j.jweia.2015.08.008.

29. F. Nicoud and F. Ducros, Flow Turbul Combust 62, 183 (1999). doi:10.1023/A:1009995426001.

30. ANSYS FLUENT Theory Guide 14.5 (2012).

31. ANSYS FLUENT User Guide 14.5 (2012).

32. C. J. Greenshields, The Open Source CFD Toolbox, Programmer's Guide, Version 3.0.1, 2015.

33. F. M. Najjar and S. Balachandar, J. Fluid Mech. 370, 101 (1998). doi:10.1017/S0022112098002110.

34. J. C. R. Hunt, A. A. Wray, and P. Moin, 1988 Summer program of the center for turbulent researchNASA Ames/ Stanford University, 1988, pp. 193. 